\title{
SEISMIC EVALUATION OF CONCRETE DAMS VIA CONTINUUM DAMAGE MODELS
}

\author{
M. CERVERA, J. OLIVER AND M. GALINDO \\ E. T. S. Ingenieros de Caminos, Canales y Puertos, Technical University of Catalonia \\ Gran Capitán s/n, Edificio C1, 08034 Barcelona, Spain
}

\begin{abstract}
SUMMARY
In this paper a general methodology for the analysis of large concrete dams subjected to seismic excitation is outlined. It is valid both for gravity dams (2D representation) and arch dams (3D representation). The method allows for non-linear material behaviour of the dam, "transparent fictitious boundaries" for properly dealing with in-coming and out-going seismic waves, and an efficient procedure to deal with dam-soil-fluid interaction. The mechanical behaviour of concrete is modelled using an isotropic damage model which allows for tension and compression damage, and exhibits stiffness recovery upon load reversals. Emphasis is placed in the treatment of fluidstructure interaction, regarding both formulation and efficiency aspects. A gravity dam and an arch dam are analysed subjected to artificially generated earthquakes of different intensities, and the results are used to study the degree of (un)safety of the dams.
\end{abstract}

\section{INTRODUCTION}

The safety evaluation of concrete dams subjected to severe seismic actions poses several difficulties to the structural analysts because it involves the solution of a complex non-linear coupled problem. The first, but not the only, difficulty is the highly non-linear mechanical behaviour of concrete in these situations, which inevitably include fracture under tension with triaxial states of stress and repeated load-unload reversals. Of course, there is a wide variety of different material models for concrete that could be used in these studies. The available literature includes models based on the theories of hypoelasticity, hyperelasticity, plasticity, fracture mechanics, plastic-fracture, or continuum damage, to name only some of the more popular ones. The present work will make use of a continuum damage model to characterize the mechanical behaviour of concrete. The Continuum Damage Theory was firstly intoduced bu Kachanov ${ }^{1}$ in the context of creep-related problems, but it has afterwards been accepted as valid alternative to deal with complex material behaviour. It is nowadays used for materials so different as metals, ceramics, rock and concrete, and 
within a wide range of applications (creep, fatigue, progressive failure, etc.). The reason for its popularity is as much the intrinsic simplicity and versatility of the approach, as well as its consistency, based on the theory of thermodynamics of irreversible processes. Among the different possibilities that such a framework offers ${ }^{2-8}$, this work will make use of an isotropic damage model, with only two scalar internal variables to monitor the local damage under tension and compression, respectively. This will provides a simple constituve model which, nevertheless, is able to capture the overall non-linear behaviour of concrete displayed under seismic loading, including the strain-softening response and the stiffness degradation and regradation observed under multiple stress reversals. Furthermore, the model can be implemented in a strain-driven form which leads to an almost closed-form algorithm to integrate the stress tensor in time. This is a most valuable feature for a model intended" to be used in large scale computations 5,9 .

Seismic analysis of a dam is a fluid-structure interaction problem. These coupled problems are of great importance invmany branches of engineering. In earthquake engineering the problem is usually argmented because in most cases the foundation must be taken into consideration, yielding a fitud-structure-soil model to be solved. This is a typical coupled problem. In fact, it is, the archetypical example of a class of coupled problems where interaction occurs at the ther the structure nor the fluidfcan be solved independently of the other: motion of the structure depends on the bydrodynamic pressures at the interface, and pressures in the fluid depend on the normal accoleration at the wet wall. It must also be considered that earthquake excitation reaches structure. This means that so -structure interaction has to be modelled in some way. In this work a block-iterative 6 chnique is used to deal with the discrete fluid-structure coupled problem ${ }^{10-13}$. This has proved to be a simple and natural approach, as well as computationally efficient, partisslarly when the non-linearity and the coupling are treated within the sane iteration loop. OBoth formulation and efficiency aspects of this approach will be briefly discussed in the following sections.

Finally, it must be remarkedrothat in these analyses both the water in the reservoir and the soil in the foundation are segi-infinite unbounded domains. For static or quasi-static loading, a fictitious boundary a sufficient large distance from the structure can be introduced in the computational बodel. The physical domain is hence "cut off", expecting that from the practical point $\bigcirc$ view, the structural response will not be affected. However, for dynamic loading this grocedure cannot be used. The fictitious boundary would reflect out-going wave back int 8 the domain of interest, spoiling completely the computed solution for any practical purp. Thus, it is neccesary to model appropriately the fictitious boundaries to allow the in coming seismic waves to enter into the domain, as well as to ensure that the out going waves are not reflected. The necessary treatment of the boundary conditions in the computational model to account for this will also be discussed below.

From the computational point of view, there are three different physical phenomena to be modelled in the seismic antalysis of a concrete dam: the structural behaviour of the dam (the final goal of the anafysis), the mechanical behaviour of the soil (both as the supporting medium of the damfand as the transmiting medium of the seismic waves), and

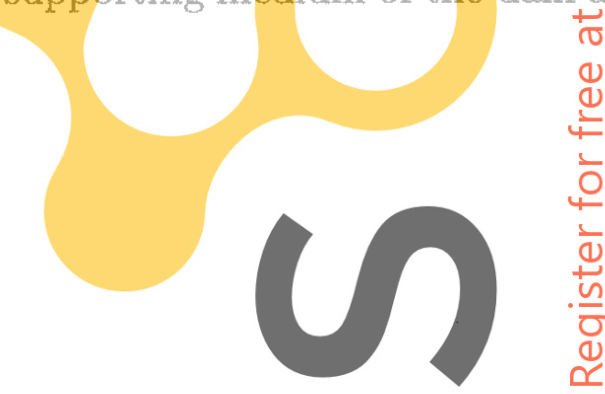




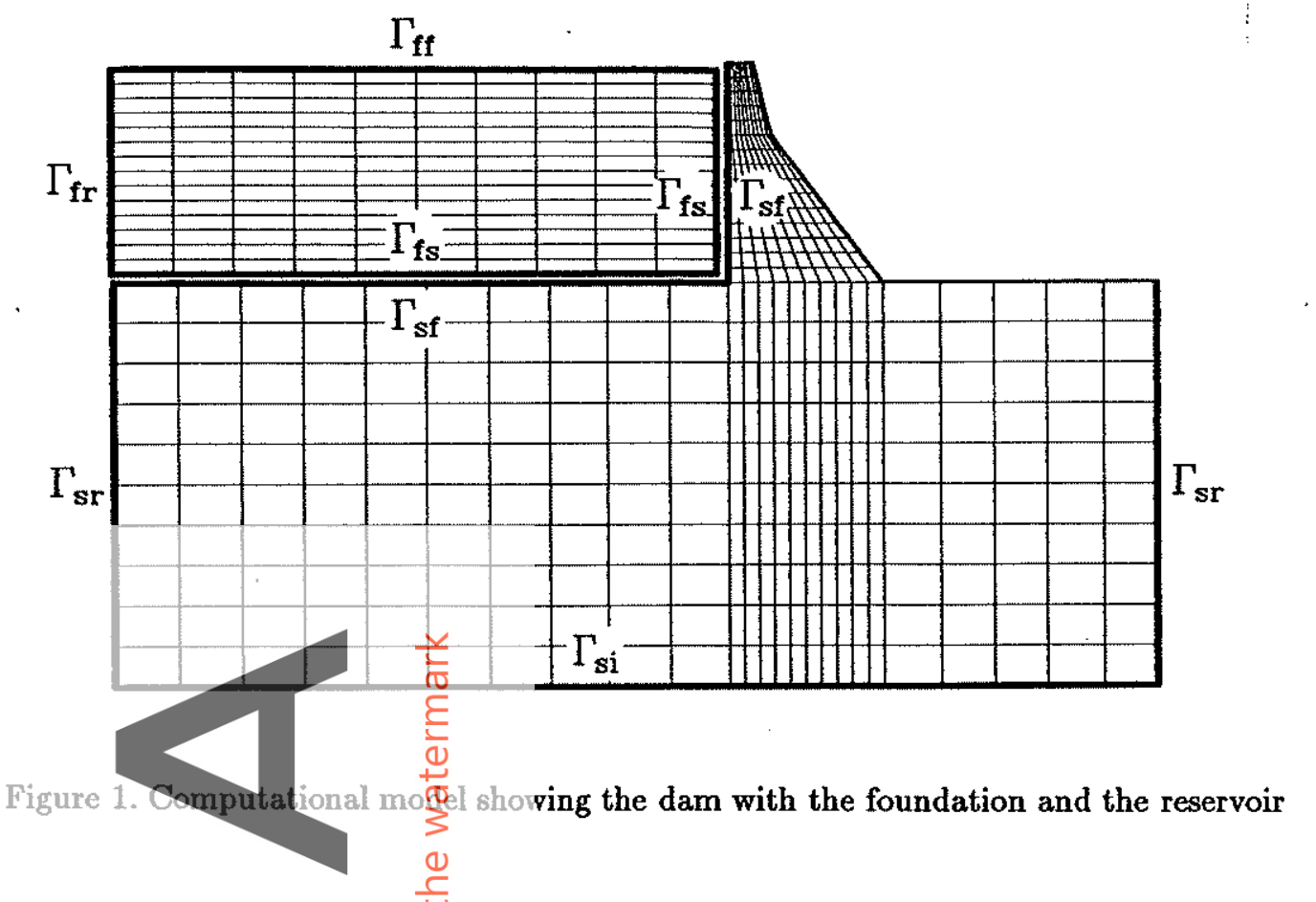

the behaviour of the water in the resvoir (as a transmiting medium of acoustic waves travelling from and towards the $\underset{\mathrm{O}}{\mathrm{p}}$-stream wall of the dam). Therefore, the computational model includes the following el eqpents (see Figure 1):

- A displacement based non-5்near dynamic model for the dam. As comented above, the non-linear behaviour is counted for here by means of a simple and computationally efficient constitutive equetion belonging to the family of the so-called 'continuum damage models'. It is al so ngcessary to include appropriate structure-fluid interaction boundaries.

- A displacoment based hinea $\underset{\mathbb{C}}{ }$ dynamic formulation for the soil. Here, 'transparent' boundaries (for out-going arflin-comming seismic waves) have to be included.

- A pressure based wave profergation formulation for the water in the reservoir. Additionaly, appropriate 'transparent' (out-going waves) radiating boundaries, fluid-soil and fluid-structure interactien boundaries and free surface boundaries need to be included.

Having these general featur $\frac{.0}{8}$ in mind, the paper is organised as follows. Section 2 describes the structural compuntional model, with especial reference to the boundary conditions and the constitutive upnodel. Section 3 describes the computational model for the fluid phase of the problem fluid-structure problem, with en some alternatives for approach the seismic evaluation of concrete dams. Sections 6 and 7 present the application of the proposed methodology to the seismic analysis of a gravity and an arch dam, respeetively. Section 8 closes the paper with some conclusions on the proposed methodology. 


\section{STRUCTURAL COMPUTATIONAL MODEL}

\subsection{Dynamic equilibrium equation}

Without going into details, the semidiscrete dynamic equilibrium equation for the structural part of the model (dam+foundation), arising from the standard Galerkin spatial discretization, reads ${ }^{15}$ :

$$
\mathbf{M}_{s} \ddot{\mathbf{a}}+\mathbf{C}_{s} \dot{\mathbf{a}}+\mathbf{S}(\mathbf{a})=\mathbf{f}_{s}
$$

where subscript $s$ refers to the solid phase in the problem. The notation involved in Eq. (1) is as follows. The mass and damping matrices are $\mathbf{M}_{\boldsymbol{s}}$ and $\mathbf{C}_{\boldsymbol{s}}, \mathbf{S}(\mathbf{a})$ is the vector of internal forces in the solid ( $\mathrm{S}(\mathrm{a})=\boldsymbol{K}_{\boldsymbol{s}} \mathbf{a}$ if a linear constitutive model is adopted, where $\mathbb{K}_{s}$ is the stiffness matrix) $\mathbf{f}_{S}$ is, the vector of external forces (including the seismic and the interaction forces, see below details), $\mathbf{a}=\mathbf{a}(t)$ is the vector of nodal displacements unknowns and the dot denotes $\mathbb{E}$ fferentiation with respect to time $t$. If the displacements u inside a finite element are integpolated in the standard way given by the shape functions $N_{s}$ and the nodal displacementşa, the expressions for matrix $\mathbf{M}_{\boldsymbol{s}}$ and vectors $\mathbf{S}(\mathbf{a})$ and $\mathbf{f}_{\boldsymbol{s}}$ are easily obtained. The dumpigg matrix accounts for both the natural viscous damping (assumed to be of Rayleigh typty and the radiation boundaries (see below for details).

2.2 Boundary conditions

In the solid domain (dam + foundation) there are three types of 'especial' boundaries to be considered in the fluid-structure problem (see Fig. 1):

(a) Solid-fluid interaction bor $\frac{1}{1}$ dary $\left(\Gamma_{s f}\right)$ : in the fluid-structure problem the coupling occurs through the interfare surface, noted here as $\Gamma_{s f}$ and $\Gamma_{f s}$ if considered as belonging to the solid or fitide fluid, respectively. Defining a unit vector $\mathbf{n}$ normal to this surface (pointing out the pressure in the fluid of be computed as,

$\mathbf{f}_{s f}=-\int_{\Gamma_{s f}} N_{f} \ln P d \Gamma=-\int_{\Gamma_{s} f} \mathbf{N}_{s}^{T} \mathbf{n} \mathbf{N}_{f} \mathbf{p} d \Gamma=-\mathbf{Q} \mathbf{p}$

where subscripts $s$ and $f$ r f्ffer to the solid and the fluid, respectively. $P$ is the pressure in the fluid, $\mathrm{P}$ is the corr shape function matrices. "The rectangular matrix $\mathbf{Q}$ is the coupling matrix. Note that if the pressure in Eq. (2) ig the total pressure, the forces will include the hydrostatic effects.

(b) Radiating or 'transparent' ary that will damp comple 3 ely the elastic waves that reach it, providing these outgoing waves are plane and onre propagating in a direction normal to the boundary ${ }^{12,14}$. This boundary provides medium, and, therefore, contribution to the damping actions which are proportional to the velocities in the effects after the spatial discretization process will be a matrix of the system that can be written as, 


$$
\mathbf{C}_{s r}=\int_{\Gamma_{s r}} \mathbf{N}_{s}^{T} \mathbf{T}^{T} \mathbf{D}_{r} \mathbf{T} \mathbf{N}_{s} d \Gamma
$$

where $\mathbf{D}_{\mathbf{r}}$ is a diagonal matrix depending on the density and the elastic constants of the solid, and $T$ is an appropriate transformation matrix depending on the orientation of the boundary. In the general case the given expression is only an approximation to a real absorbing boundary, but numerical experiments show that the error involved in this approximation is acceptable from an engineering point of view.

(c) Radiating boundary with incoming wave $\left(\Gamma_{s i}\right)$ : the fictitious boundary at the bottom of the model must be able to damp the out-going elastic waves and, at the same time, be able to allow the prescribed in-coming seismic waves to enter the domain. Under the same assumptions that for the previous case, the corresponding boundary will contribute to the tamping matrix and to the vector of nodal forces. The

$$
\begin{aligned}
& \underbrace{}_{s i}=\int_{\Gamma_{s i}} \mathbf{N}_{s}^{T} \mathbf{T}^{T} \mathbf{D}_{r} \mathbf{T} \mathbf{N}_{s} d \Gamma \\
& \qquad \int_{\Gamma_{s i}} \mathbf{N}_{s}^{T} \mathbf{T}^{T} \mathbf{D}_{\mathbf{r}} \mathbf{T} \dot{v} d \Gamma
\end{aligned}
$$

where $\dot{v}$ is the prescribed iin coming velocity wave. Note that in this way the seismic input is introduced as a '" velocities. This is different from the traditional approach, where the seismic input is prescribed as a forced'. Gondition, in terms of the ground accelerations. The advantage is that now we areable to write Eq. (1) in terms of the total displacements, velocities and acceierations in the structure, rather than 'relative' to the ground. The formulation is clearer in the way, and note that there will be no 'forced' boundary conditions (prescribed disptacements) in the structural model.

Note that we have made exp $\frac{O}{\text { f }}$ cit the expressions for the nodal forces due to the water and the seismic input. Apart from these the vector $\mathbf{f}_{\boldsymbol{s}}$ in Eq. 1 will typically contain only the system of self weight nodal forces. It may also be remarked that in many applications is not necessary to make use of the fictitious boundaries of type (b). If the portion of foundation included in the comptational mesh is large enough, these can be substituted by a 'repeatibility condition', plescribing the displacements in the left-tiand side of the model to be equal to those in right-hand side ${ }^{12,25}$. This is satisfactory for most of 2D and many 3D applications, @- though naturally this will always depend on the actual geometry of the problem.

\subsection{Discretization in time. Algo thmic and natural damping}

Due to the non-linearity introdwed in the computational model through the constitutive equation for concrete, the internf force vector $\mathbf{S}(\mathbf{a})$ is a time-history dependent quantity. Therefore, the dynamic equilibrivm equation (1) must be solved in the time domain, following a step-by-step procedure There are several methods in the literature available for 
the discretization and integration in time of such second order equations. In this work we will make used of the well-known and widely employed Newmark method, but others equally suitable would be the SS22 method, the $\alpha$-method, or the Houbold's or Park's methods ${ }^{15,16}$. Each of these methods has its own characteristics regarding stability and accuracy. It must also be remarked that because of the non-linear nature of Eq. (1) it will be most convenient to use any of the previous methods in a predictor-multicorrector form, using a scheme such as the Newton-Raphson method to linearize the equations for each time step of the time integration process ${ }^{12,17}$.

Because the higher modes of semidiscrete structural equations are spurious subproducts of the discretization process and not representative of the governing partial differential equations, it is generally viewed as desirable, and often is considered absolutely necessary, to have some form of algorithmic damping present to remove the participation of the high-frequency modal components. This is even more crucial in non-linear analysis ${ }^{18}$. In terms of the Newmark methyd, the parameters $\gamma$ and $\beta$ determine the characteristics of stability and scuracy of themethod. It is necessary to chose $\gamma>1 / 2$ to introduce high-frequency dissipation. For $\frac{}{2}$ fixed $\gamma$ one can chose $\beta=(\gamma+1 / 2)^{2} / 4$ to maximize this dissipation while retaining uncere ditional stability. However this leads to a- drop to first order accuracy of the method. Fis is not acceptable, the $\alpha$-method can be used, which can introduce algorithmic dissifation while retaining unconditional stability and second

order accuracy 16,18 . considered in the constitutive mol for concrete. However, it will be seen that this does not contemplate energy dissipation in a load-unload-reload cycle, as it would be desiderable under seismic excitation. This a ded damping effect mat be heuristically considered via the damping matrix $\mathrm{C}_{\xi}$. Due to the fack of experimental results on the damping mechanisms of mass concrete dams under seismitc loading we will follow the usual finite element procedure of assuming a Paylcigh type of $\frac{d}{+}$ amping, i.e.,

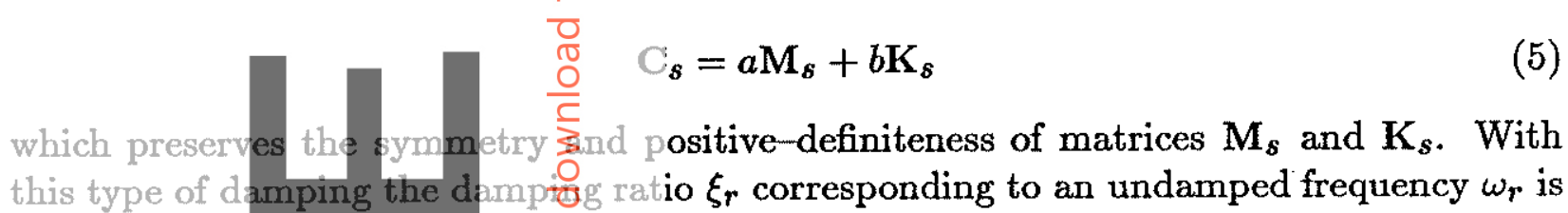

$$
\xi_{r}=\frac{1}{2}\left(\frac{a}{\omega_{r}}+b \omega_{r}\right)
$$

The constant $a$ and $b$ are calis

Eq. (6) that the effect of the "m

the 'stiffness damping" will sev

the computational model a sign

out the high-frequency compon

occurs inside the dam the stiffi

the stiffness matrix in Eq. (5)

or secant matrix 18 . The questor

computational efficiency we wil

rated to provide the desired amount of damping in two

cally $3-10 \%$ in the first and second modes. It is clear from 엉s damping' will be neglegible for the higher modes, while Nely damp them. It is therefore desiderable to include in

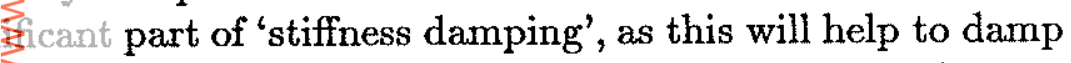
ents of the response. It has to be remarked that as damage will also be degradated. Thus, it may be discussed if Should be the initial (elastic) one, or an updated tangent

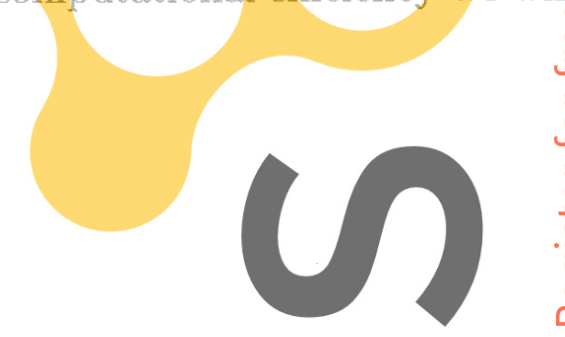




\subsection{Constitutive model for concrete}

The Continuum Damage Mechanics Theory is based on the definition of the effective stress concept, which is introduced in connection with the hypothesis of strain equivalence ${ }^{2}$ : the strain associated with a damaged state under the applied stress $\sigma$ is equivalent to the strain associated with its undamaged state under the effective stress $\bar{\sigma}$. In the present work the effective stress tensor $\overline{\boldsymbol{\sigma}}$ (second order) will assume the following form:

$$
\overline{\boldsymbol{\sigma}}=\mathbf{D}_{0}: \boldsymbol{\epsilon}
$$

In this expression $\mathbf{D}_{\mathbf{0}}$ is the usual fourth order linear-elastic constitutive matrix, $\boldsymbol{\epsilon}$ is the second order strain tensor, and (:) denotes the tensorial product contracted on two indices. As our aim is to use a scalar damage model, with separated internal damage variables for tensile and compressive stress contributions, a split of the effective stress tensor into tensile and compressive componts is needed. In order to clearly identify contributions respecting to each on of these Endependent effective stress tensors, $(+)$ and $(-)$ indices will be extensively used, referring to tensile and compressive entities, respectively. In this work, the stress split will be pers

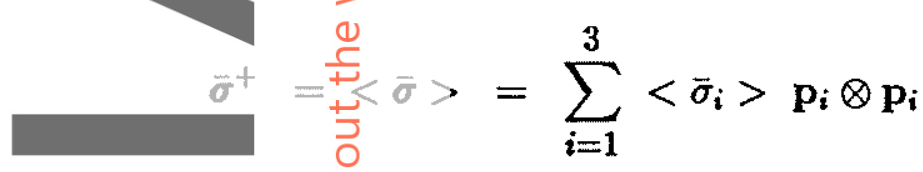

$$
\begin{aligned}
& =\sum_{i=1}^{3}>\bar{\sigma}_{i}<\mathbf{p}_{i} \otimes \mathbf{p}_{i}
\end{aligned}
$$

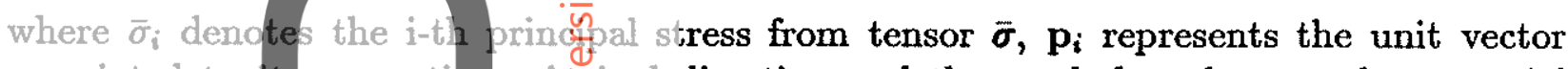
associated to its respective prirteipal direction and the symbol $\otimes$ denotes the tensorial product. The symbols $\langle\cdot>$ ate the MacAuley brackets (thus giving the value of the enclosed expression when positive, and setting a zero value if negative), and symbols $>$. < are such that

Hence, according to this stress splitting, the contitutive law proposed in this work can be explicitely defined ${ }^{19}$, renderilos for the Cauchy stress tensor $\boldsymbol{\sigma}$ the final expression:

$$
\sigma \rightleftharpoons\left(1-d^{+}\right) \overline{\boldsymbol{\sigma}}^{+}+\left(1-d^{-}\right) \overline{\boldsymbol{\sigma}}^{-}
$$

with

and

$$
0 \leq d^{-} \leq 1
$$

where $d^{+}$and $d^{-}$are the tensil, fund compressive damage variables, respectively. Thermodynamic considerations aboun the non-negativeness of the dissipation ${ }^{19}$ demand that $\dot{d}^{+} \geq 0$ and $\dot{d}^{-} \geq 0$. The modes is completed with appropriate evolution laws for these internal damage variables.

In order to clearly define conchtts such as loading, unloading, or reloading, a scalar positive quantity, termed equivalen $\frac{1}{3}$ tress, will be defined. This enables to compare different tridimensional stress states. With such a definition, distinct tridimensional stress states can be mapped to a single equ药alent unidimensional stress test, which makes possible

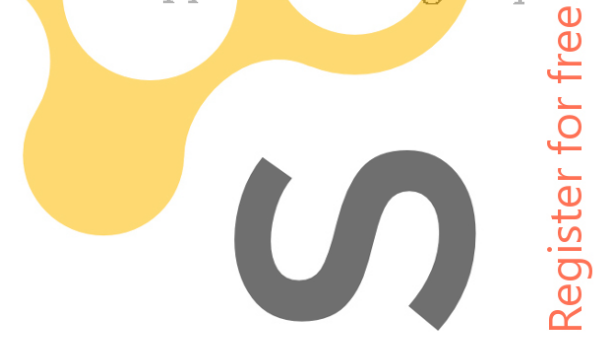


their quantitative comparison. As a consequence of the stress split, an equivalent effective tensile norm $\bar{\tau}^{+}$and an equivalent effective compressive norm $\bar{\tau}^{-}$will be used. In the present work they will assume the following forms:

$$
\begin{aligned}
& \bar{\tau}^{+}=\sqrt{\overline{\boldsymbol{\sigma}}^{+}: \mathbf{D}_{\mathbf{0}^{-1}: \overline{\boldsymbol{\sigma}}^{+}}}, \\
& \bar{\tau}^{-}=\sqrt{\sqrt{3}\left(K \bar{\sigma}_{\text {oct }}^{-}+\bar{\tau}_{o c t}^{-}\right)}
\end{aligned}
$$

In Eq. (10.b) $K$ is a material property which depends on the ratio between the uniaxial and biaxial compressive strengths of the concrete ${ }^{19}$ (a typical value for concrete is $K=0.17$ ); additionally, $\bar{\sigma}_{\text {oct }}^{-}$and $\bar{\tau}_{\text {oct }}^{-}$are, respectively, the octhaedral normal stress and the octhaedral shear stress, obtained from $\bar{\sigma}^{-}$.

With the above definitions $f_{9}$ the effective equivalent norms, two separated damage criteria $g^{+}$and $g^{-}$will beintrod ${ }^{\frac{2}{*}} d^{4}$, the former for tension and the latter for compression:

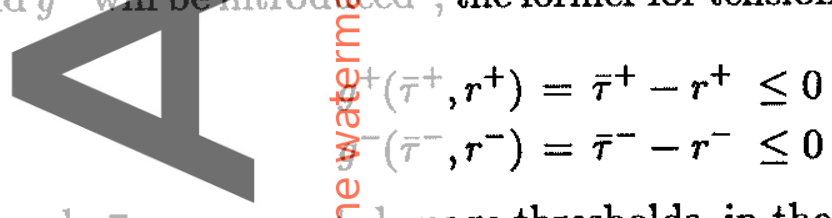

Variables $r^{+}$and $r^{-}$are current darnage thresholds, in the sense that their values control the size of the expanding damatre suraces. For the initial stage, that is, when no loading has yet been applied, values $r_{0}^{\oplus}$ and $r_{0}^{-}$, assumed material properties, are attributed to these thresholds. As it can deduced from definitions (10.a) and (10.b), Eq. (11.a) corresponds to a damage bounging surface which is a sphere centered at the origin in the space of principal undanaged Ensile stresses ${ }^{9}$, and Eq. (11.b) defines a Drucker-Pragger cone for compression. Eq. (11. (d) states that tensile damage tends to increase if $\bar{\tau}^{+}=r^{+}$, and so it will be initiated when for the first time $\bar{\tau}^{+}=r_{0}^{+}$(a similar reasoning can be applied for compression). Figwe 2 shows the initial damage bounding surface resulting from the combination of both eriteria in a biaxial effective principal stress space. Note the good qualitative agreement witg the documented experimental results for concrete.

With these definitions, and fifter enforcement of the consistency conditions for loadingunloading-reloading situations ङ्रia the Kuhn-Tucker relations, the kinematics of the tensile and compressive internal varialiges are defined as:

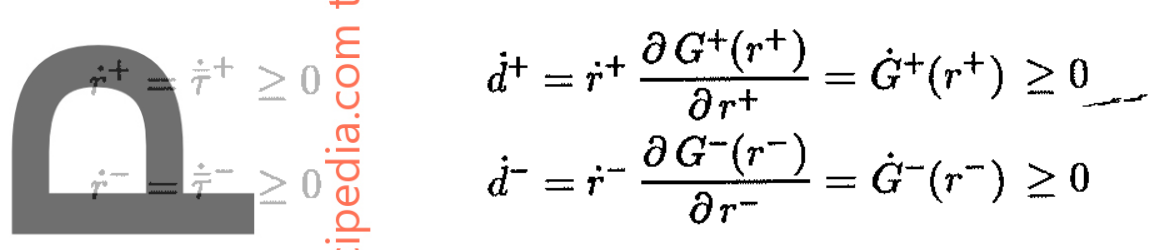

with $G^{+}$and $G^{-}$being approyfiate monotonically increasing functions derived from experimental observation. The particular forms assumed by the rate Eqs. (12.a) and (12.b) (with the initial condition of not danages):

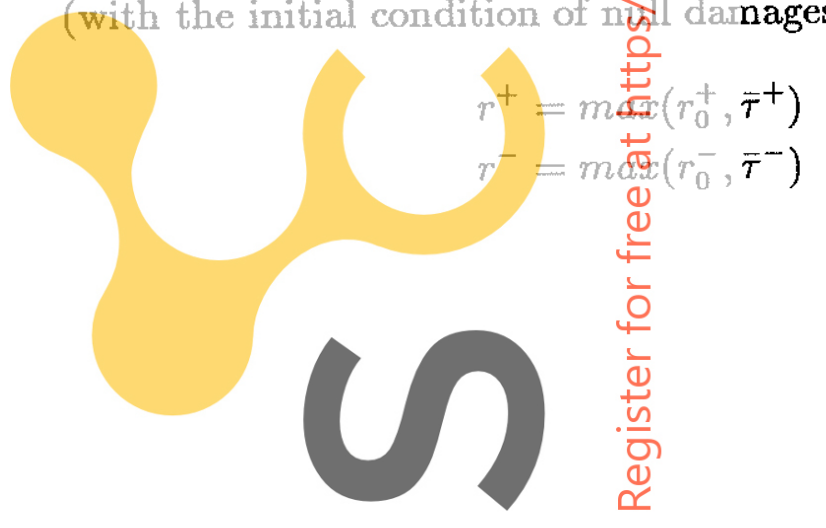




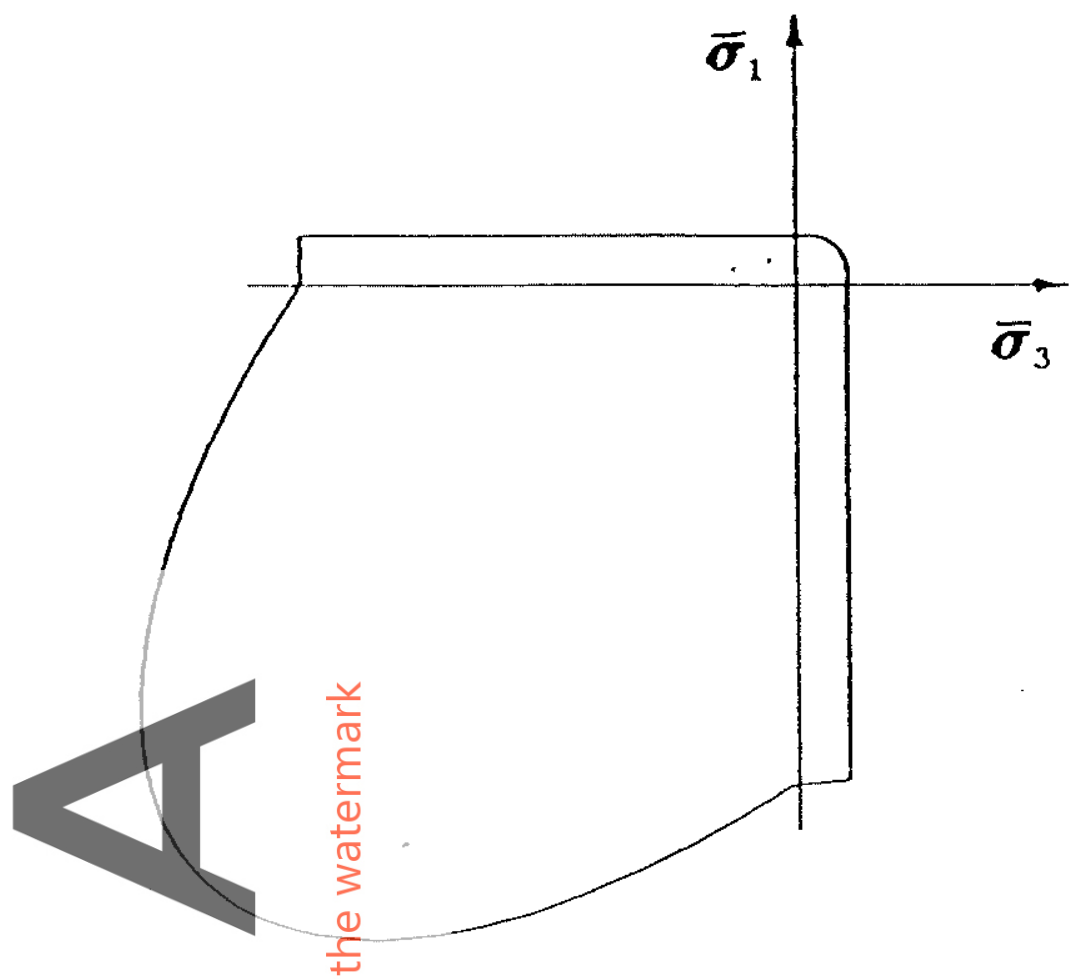

Figure-2. Initial damage botyding surface in a biaxial effective principal stress space

These equations putinto evilence that the model is strain driven in a closed form: once the current strain tensor known, damage variables can be easily evaluated, as they only depend on the equivent norms $\bar{\tau}^{+}$and $\bar{\tau}^{-}$, which are evaluated from $\epsilon$. So, the selection for the particular damage evolutions to be consid $\mathcal{y}^{\mathrm{C}} \mathrm{d}$, and consequently some care must be devoted to this subject, so that a realistic rep Anyway, the change from one peinticular set of evolution laws to a different one does not put any special problem, thus enting this model to have substantial updating versatility.

For the present work, the following damage evolution rules will be adopted:

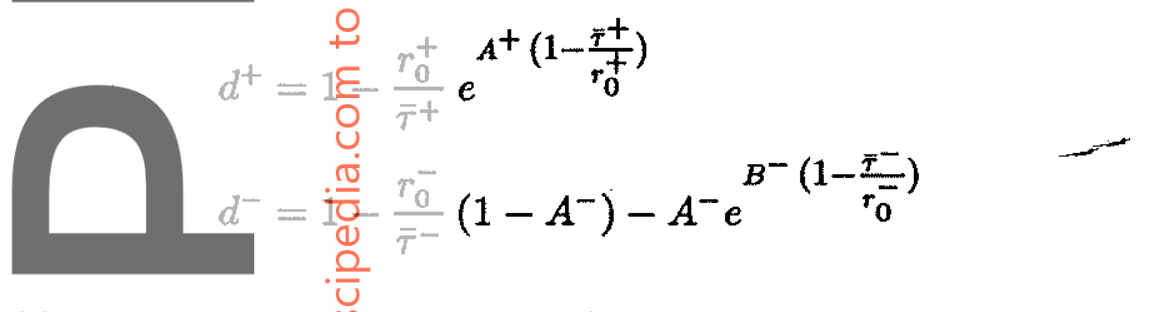

Eq. (14.a) is able to reproduce we softening branch of a concrete unidimensional tensile test, asymptotically to the strain axis. With this evolution law for $d^{+}$, a finite area is retained between the stress-strain $u r v e$ and the strain axis, which is crucial to appropriately define the fracture energy concent (as it is well known, of primary importance to satisfy requisits of mesh-objectivity when dealing with softening materials) ${ }^{9}$. Besides the tensile damage threshold $r_{0}^{+}$(usually reftated to the uniaxial tensile peak strength), only the parameter $A^{+}$exists, the determirlation of which is made by equating the material fracture

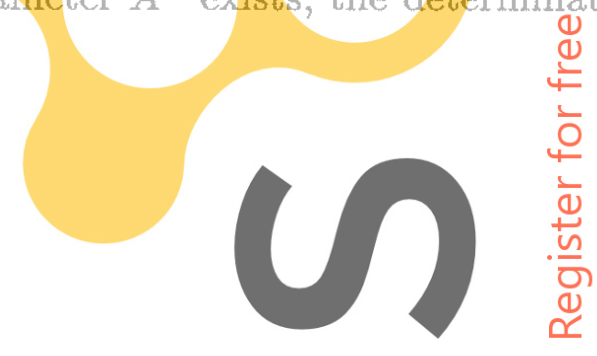


energy to the time integral of dissipation ${ }^{19}$. By means of Eq. (14.b) it is possible to reproduce the hardening effect on concrete submitted to compression, as well the softening wich occurs after the compressive strength is attained ${ }^{8}$. Besides $r_{0}^{-}$, for its characterization two parameters $\left(A^{-}, B^{-}\right)$must be defined, usually by imposing that the evolution curve satisfies two selected points of a unidimensional experimental test ${ }^{19}$.

It must be remarked that the aim of this model is to be able to model properly the stiffness degradation of concrete both in tensile and compressive processes. Moreover, the introduction of independent internal damage variables for tension and compression allows for stiffness recovery (also known as unilateral effect) upon load reversal, a feature which the authors have found of primary importance when conducting seismic analysis of structures. Figure 3 shows the cyclic behaviour of the proposed continuum damage model in a uniaxial test.
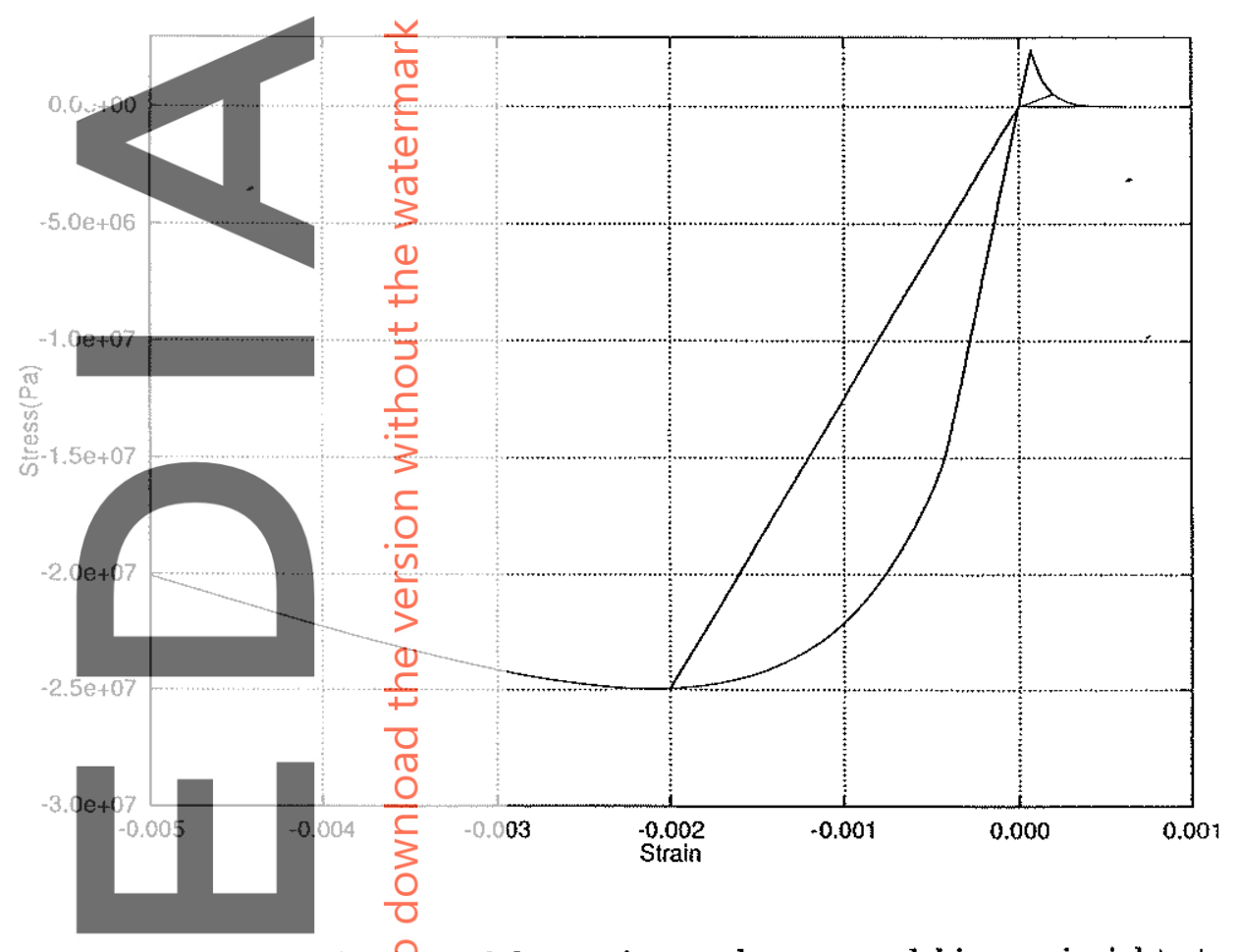

Figure 3. Cyclic behaviour of the continuum damage model in a uniaxial test

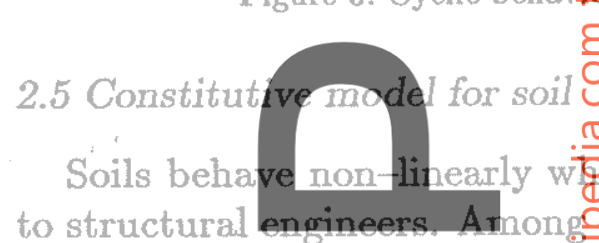

- _constitutive modelling, one of the alternatives is to use a similar constitutive model to that used for the structure, that is, continuum damage model with appropriate parameters. However, when detcrmining thinput seismic excitation starting from the motion of one free-field surface control point, the principle of superposition is frequently invoked. This means that a linear soil systerfis actually assumed. The simplification of using a linear soil medium should be considered in connection with the actual way of determining and specifying the seismic input.

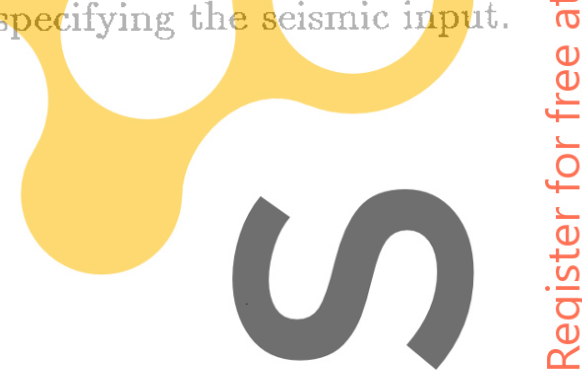




\section{FLUID COMPUTATIONAL MODEL}

\subsection{Dynamic governing equation}

The governing equation for acoustic waves for an inviscid, compressible fluid with small amplitudes for the displacements and velocities is the well-known Helmholtz equation:

$$
\nabla^{2} P-\frac{1}{c^{2}} \ddot{P}=0
$$

where $P$ is the pressure and $c$ is the acoustic speed in the fluid. After the standard process of discretization in space ${ }^{15}$ the semidiscrete governing equation can be written as

$$
\mathbf{M}_{f} \ddot{\mathbf{p}}+\mathbf{K}_{f} \mathbf{p}=\mathbf{f}_{f}
$$

where subscript $f$ refers to the fluid. The 'mass' matrix is $\mathbf{M}_{f}, \mathbf{K}_{f}$ is the matrix arising from the discretization of the (linear) Laplace operator, $\mathbf{f}_{f}$ is the vector of 'force', and $\mathbf{p}=\mathbf{p}(t)$ is the vector of nodal pressures in the fluid. Note that only natural boundary conditions have been considered and that no viscous damping term is present in Eq. (16). Note also that this is a second order equation in time, and so it can be integrated stepby-step in an analogous manner to Eq. (1).

\subsection{Boundary conditions}

In the fluid domain (water in the reservoir) there are three types of 'especial' boundaries to be considered in the fluid-structure problem (see Fig. 1):

(a) Solid-fluid interaction boundary $\left(\Gamma_{f s}\right)$ : in the fluid-structure problem the normal velocities in the fluid (or their time derivatives) are prescribed to be equal to those in the solid. It can be shown ${ }^{15}$ that this condition leads to a prescription on the derivative of the pressure normal to the interface:

$$
\nabla P \cdot \mathbf{n}=\rho_{f} \ddot{\mathbf{u}} \cdot \mathbf{n}
$$

where $\mathbf{n}$ is a unit vector normal to the interface (pointing inwards to the fluid), $\rho_{f}$ is the fluid density and $\ddot{\mathbf{u}}$ is the acceleration. After discretization, this condition appears as a term of nodal forces on the fluid due to the accelerations in the solid that can be computed as,

$$
\mathbf{f}_{f s}=\rho_{f} \int_{\Gamma_{f s}} \mathbf{N}_{f}^{T}(\mathbf{n} \cdot \ddot{\mathbf{u}}) d \Gamma=\rho_{f} \int_{\Gamma_{f s}} \mathbf{N}_{f}^{T} \mathbf{n}^{T} \mathbf{N}_{s} \ddot{\mathbf{a}} d \Gamma=\rho_{f} \mathbf{Q}^{T} \ddot{\mathbf{a}}
$$

Note that the coupling matrix $\mathbf{Q}$ is the same as in Eq. (2).

(b) Radiating or 'transparent' boundary $\left(\Gamma_{f r}\right)$ : For the fluid domain it is also possible to derive a fictitious boundary that will damp completely the pressure waves that reach it, in a way similar to the one described for the solid, and under the same 
assumptions $^{12,14,15}$. It can be shown that this condition leads to a prescription on the derivative of the pressure normal to the interface of the form:

$$
\nabla P \cdot \mathbf{n}=-\frac{1}{c} \dot{P}
$$

where $\dot{P}$ is the time derivative of the pressure. This boundary provides tractions which are proportional to the velocities in the fluid, and therefore, its effects after the spatial discretization process will be a contribution to the damping matrix of the system that can be written as,

$$
\mathbf{C}_{f r}=\frac{1}{c} \int_{\Gamma_{f r}} \mathrm{~N}_{f}^{T} \mathrm{~N}_{f} d \Gamma
$$

For some applications it would be handy to have a 'transparent' boundary for the fluid with in-coming seismic gave $\left(\Gamma_{f i}\right)$. This is easy to derive based on the boundary just described. The only difference is an added 'force' term which depends on the prescribed seismic velocities $\dot{v}$, and which reads:

$$
\mathbf{f}_{f i}=\frac{2}{c} \int_{\Gamma_{f i}} \mathbf{N}_{f}^{T} \dot{v} d \Gamma
$$

(c) Free surface boundary $\left(\Gamma_{f f}\right)$ : On the free surface of the fluid the simplest assumption is that

$$
P=0
$$

and this can be imposed as a 'forced' condition at equation solution level. If the possibility of having surface gravity waves is considered, this can be substituted by the linearized surface wave condition ${ }^{15}$, which results in an addition to the 'mass' matrix of the form

$$
\mathbf{M}_{f f}=\frac{1}{g} \int_{\Gamma_{f f}} \mathrm{~N}_{f}^{T} \mathrm{~N}_{f} d \Gamma
$$

where $g$ is the gravity acceleration.

\section{FLUID-STRUCTURE INTERACTION}

\subsection{Governing equations and direct solution}

Doubtless, fluid-structure interaction is one of the best known coupled problems in engineering. In this case, the coupling occurs at the interface between two different domains, occupied one by the solid and the other by the fluid. Neither the structure nor the fluid 
can be solved independently of each other, since the motion of the structure depends on the pressures of the fluid at the interface, and the fluid pressures depend in turn on the normal acceleration of the wet wall of the solid. The equations considered here are the conservation of momentum for the solid (see Section 2) and the Helmholz equation for the fluid (see Section 3), both equations being coupled through the corresponding (moving) interface boundary terms. The constitutive relationship adopted for the structure is the nonlinear isotropic continuum damage model described in Section 2, whereas a linear elastic model is assumed for the foundation.

The semidiscrete problem arising from the standard Galerkin spatial discretization reads as follows:

$$
\begin{aligned}
\mathbf{M}_{s} \ddot{\mathbf{a}}+\mathbf{C}_{s} \dot{\mathbf{a}}+\mathbf{S}(\mathbf{a}) & =\mathbf{f}_{s}-\mathbf{Q} \mathbf{p} \\
\mathbf{M}_{f} \ddot{\mathbf{p}}+\mathbf{C}_{f} \dot{\mathbf{p}}+\mathbf{K}_{f} \mathbf{p} & =\mathbf{f}_{f}+\rho_{f} \mathbf{Q}^{T} \ddot{\mathbf{a}}
\end{aligned}
$$

where subscripts $s$ and $f$ refer to the solid and the fluid, respectively, and the coupling forces have been explicitely written. Remember that the coupling matrix $\mathbf{Q}$ only has non zero terms for the nodes located on the wet wall. Observe that in Eq. (24) the coupling is linear and the only non-linearity of the problem comes from the constitutive model for the solid (the dam). Therefore, after using a suitable time discretization (the Newmark scheme, for example) one will be led to a non-linear algebraic system of the form:

$$
\left[\begin{array}{cc}
\mathbf{A}_{s s}(\mathbf{a}) & \mathbf{A}_{s f} \\
\mathbf{A}_{f s} & \mathbf{A}_{f f}
\end{array}\right]\left[\begin{array}{l}
\mathbf{a} \\
\mathbf{p}
\end{array}\right]=\left[\begin{array}{c}
\overline{\mathbf{f}}_{s} \\
\overline{\mathbf{f}}_{f}
\end{array}\right]
$$

This system of equations can be easily symmetrized by scaling properly the equation for the fluid pressure, and the coupled problem can be directly solved, in a step-by-step fashion, by forming and solving Eq. (24) step after step. Iterations would be needed within each step due to the non-linearity of the term $\mathbf{A}_{s s}(\mathbf{a})$. One disadvantage of this strategy is that the structure of the global matrix of the system is such that entries come from the two different domains (solid and fluid), and so, integrals have to be evaluated in two different domains, and they represent physically different magnitudes. Another disadvantage is the larger size of the global matrix as compared with the ones arising from the different domains. On the other hand, the advantage is that the final algorithm is easily and clearly defined, and its analysis, regarding for instance stability, is feasible (although complicated).

\subsection{Block-iterative solution}

Let us consider now the use of block-iterative algorithms to solve problem (24). This will reduce the size of the resulting subproblems at the expense of iterating. Assuming that the first equation in (24) is solved first, there are two possible block-iterative schemes, namely,

$$
\begin{aligned}
\mathbf{A}_{s s}\left(\mathbf{a}^{(i)}\right) \mathbf{a}^{(i)} & =\overline{\mathbf{f}}_{s}-\mathbf{A}_{s f} \mathbf{p}^{(i-1)} \\
\mathbf{A}_{f f} \mathbf{p}^{(i)} & =\overline{\mathbf{f}}_{f}-\mathbf{A}_{f s} \mathbf{a}^{(k)}, k=i-1 \text { or } i
\end{aligned}
$$

Here, superscripts in parenthesis refer to iteration counters. For $k=i-1$ this is the block-Jacobi (or block-total-step) method, whereas for $k=i$ it is the block-Gauss-Seidel 
(or block-single-step) method. From elementary numerical analysis it is known that, under certain conditions, both the Jacobi and the Gauss-Seidel methods converge linearly when applied to linear systems, the convergence rate of the latter being twice higher than that of the former. In their block counterparts these properties are inherited, the convergence rate depending now on the spectral radius of the matrices involved.

On the other hand, problem (25) (and also problem (26)) is non-linear, so that an iterative procedure must be used to deal with this non-linearity. Both the Picard (or fixed point) and the Newton-Raphson methods are suitable candidates to perform the necessary linealization. Either the non-linearity or the coupling could be dealt with in two nested iterative loops. However, there is the strong temptation to use a single iterative loop to deal both with the non-linearity and the coupling ${ }^{10,11,13}$. This would lead (for the Picard method) to:

$$
\begin{aligned}
\mathbf{A}_{s s}\left(\mathbf{a}^{(i-1)}\right) \mathbf{a}^{(i)} & =\overline{\mathbf{f}}_{s}-\mathbf{A}_{s f} \mathbf{p}^{(i-1)} \\
\mathbf{A}_{f f} \mathbf{p}^{(i)} & =\overline{\mathbf{f}}_{f}-\mathbf{A}_{f s} \mathbf{a}^{(k)}, k=i-1 \text { or } i
\end{aligned}
$$

Perhaps the simplest choice to implement is: once $\mathbf{a}^{(i)}$ is known by solving Eq. (27), it is used to evaluate the right-hand side (RHS) of Eq. (28), and then this can be solved.

Box 1 presents the computational algorithm one of these block-iterative techniques applied to the solid phase of the fluid-structure interaction problem. Note that here an implicit predictor-multicorrector scheme ${ }^{11,17}$ has been assumed for the solution of the non-linear transient problem, without loss of generality. Obviously, the computational algorithm for the fluid phase could follow an identical or similar procedure. The squares labelled "FLUID" refer to the necessary inter-communication to interchange data between the solid and the fluid phases in the problem. Note also the modify/unmodify steps introduced for the evaluation of the residual force vector, which save unnecessary reevaluations of the internal forces $\mathbf{S}(\mathbf{a})$ every time that the coupling term is recalculated.

The advantage is now that the structure of the matrices in the left-hand side, $\mathbf{A}_{s s}$ and $\mathbf{A}_{f f}$, is such that entries come only from the single field currently considered, and so, integrals have to be evaluated only in that domain, and they represent physically homogeneous magnitudes. Moreover, for many practical applications these matrices are symmetric. There is no difficulty in evaluating the coupling terms appearing in the RHS of Eqs. (27) and (28), as they only involve integrals over the 'interaction boundary', and this is "seen" from both domains. Finally, note that the two systems of equations to be solved are smaller in size and with reduced band-width, as well as better conditioned, as compared to that yielding from the direct solution of Eq. (25). On the other hand, the disadvantage of the block-iterative solution of Eq. (24), is that iterations will be needed even if the problem is linear (note the need to check global or overall convergence in Box 1). This is not especially inconvenient if the problem is non-linear, as equilibrium iterations would be required anyway, or if the coupling effect is not too strong.

As the time dimension is involved, the analysis of any proposed solution strategy must consider the time integration stability of the approach. It may happen that, even if an unconditionally stable algorithm has been used for every one of the fields, the overall algorithm may still be conditionally stable ${ }^{10,15}$. In practice, as the size of the chosen time step will always be limited by accuracy considerations, this may not be a serious 


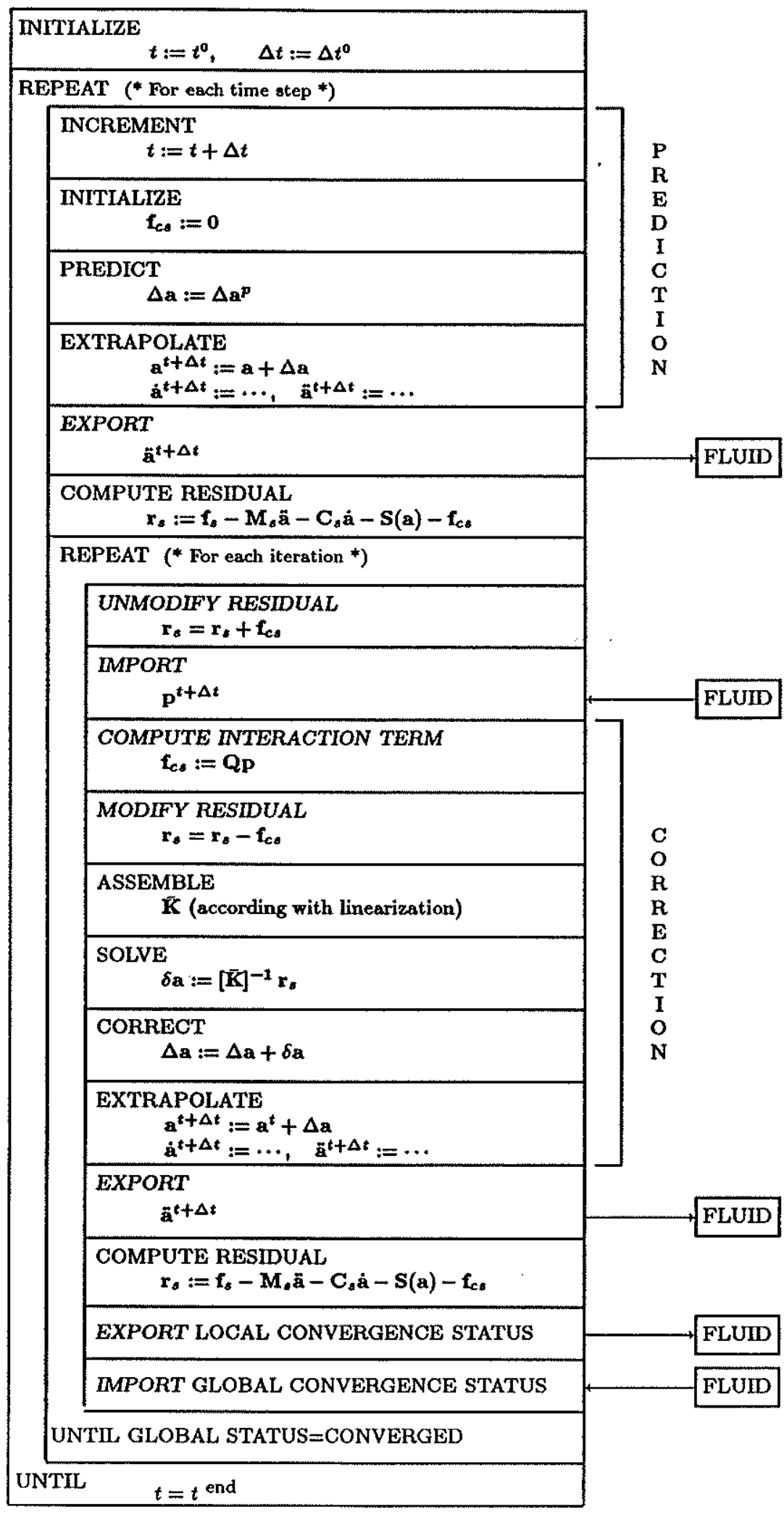

Box 1. Computational algorithm for block-iterative technique applied to the solid phase of the fluid-structure interaction problem. 
drawback. Regarding the stability analysis of the block-iterative solution, it is certainly complicated and very problem dependent. Stabilization methods have been proposed to ensure unconditional stability of the block-iterative solution for specific problems under certain circumstances $15,21,22$, but they require matrix operations that destroy the modularity (and so, the main motivation) of this approach.

An important point to remark regarding stability of this iterative technique is that time integration stability will also depend on the tolerance demanded to achieve overall convergence. If the solution of problem (26) is iterated until full overall convergence is achieved, then the stability characteristics of the approach are identical to those of the direct solution (problem (25)). However, as this is impossible or impractical to manage in real computations, a new source of instability will come from the tolerance specified by the user. As a limit case, if no check on the overall convergence is made, the approach becomes block-explicit (also known as "staggered" methods), and it will be obviously conditionally stable, or, in some unfortunate cases, unconditionally unstable ${ }^{10,12,23}$. Sensibility to this factor is again very problem dependent. No difficulty was found by the authors in the numerical examples run for fluid-structure interaction even if slack tolerances were used.

The main conclusion from the authors' experience in solving this and other coupled problems is that dealing with the non-linearity and the coupling within a single iterative loop is very effective. Roughly speaking, the number of iterations due to the nonlinearity and the coupling do not add up. Even with weak nonlinearities, these are who govern the global process, so that the coupling is achieved automatically, and unexpensively ${ }^{13}$. For a more detailed discussion of the efficiency of the block-iterative techniques applied to coupled problems, as well as some clues to their implementation, see reference 24 .

\section{SEISMIC EVALUATION OF CONCRETE GRAVITY DAMS}

When dealing with a large structure it is important to be able to quantify somehow a 'factor of safety' to measure the safety of the structure against the possibility of failure. This is not a simple concept to apply in cases of complex non-linear structural behaviour, as present, for instance, in the seismic analysis of dams. The difficulties associated to this and similar definitions have been clearly stated by Fanelli ${ }^{26}$ : “... the currently accepted quantitative definitions of the 'factor of safety' of a dam are rather fuzzy concepts, open to criticism from a rational viewpoint... In each instant of a dam's life, there will be a definite relationship between the 'load' acting on it and the 'ultimate load' of the same type that the dam is able to withstand (this concept of 'ultimate load' requires that the path to 'failure' and the very concept of 'failure', be defined in an unambiguous way, which is by no means a trivial operation). ... [The factor of safety] is a continuously changing function of time, as load vary and the dam ages; its instantaneous physical values cannot be computed in a realistic way, because we lack complete information on loads and material properties and distribution. Besides, these values are also bound to vary considerably according to the different definitions of the 'path to failure' for any given situation. ... So, we are forced to conclude that there is not, in any given instant, a unique value of the safety factor, but rather and indefinite number of them, and that all those safety factors are also continuously varying in time". 
Additionally, there are situations in which the occurrence of a certain degree of 'local failure' is unavoidable. Such is the case of a concrete dam subjected to severe seismic excitation, where stress reversals will almost certainly produce damage in certain areas of the dam. This will not, in general, lead to the (global) collapse of the structure. In some rare cases, the non-linear behaviour of the material and the change of stiffness associated to partial damage may even help the structure to withstand the earthquake without reaching a state of 'failure'.

Nevertheless, the concept of safety (or unsafety) of a structure is intuitively linked to the 'damage' that it suffers when subjected to a particular external action. The 'ultimate load' that a structure is able to withstand is associated to a high level of damage, while a load producing little or no damage is far from leading to structural collapse. Keeping in mind that there may exist mechanisms of global collapse not associated to damage (elastic inestability, brittle fracture, etc.) the present work intends to study the intuitive relation between damage and safety.

\subsection{The concept of global damage}

The damage or degradation variables $d^{+}$and $d^{-}$defined in Section 2 are a measure of the loss of secant stiffness of the material at a point and a at given time. Therefore, these variables are understood as a measure of the 'local damage' inside a structure, and as such it provides very useful information to the analyst. The purpose of this section is to introduce a 'global damage index' $D^{+}$(and $D^{-}$) whose value gives an indication of the state of the structure, as a whole, at a given time.

Some important aspects must be considered for the definition of such a global damage index: (i) The global damage index must be the same for two identical structures with the same distribution of local damage, regardless of the instant of time or the type of loading. Therefore, the global index must be computed uniquely in terms of the values of the local damage variable, defined at each point inside the dam, and its distribution; (ii) As the local variable $d^{t}$, the global index $D^{t}$ must be a non-dimensional scalar value, ranging between 0 , for an undamaged structure, to 1 , for a fully degradated structure; (iii) The structure is undamaged $\left(D^{t}=0\right)$ if and only if no local damage has occurred $\left(d^{t}=0\right.$ for all points); similarly, the structure is fully degradated $\left(D^{t}=1\right)$ if and only if all the point are fully damaged ( $d^{t}=1$ for all points); (iv) If a structure with a certain degree of damage is subjected to an external action, the global damage index can only increase or remain unchanged.

Thus, we will define the global damage index for a structure, at a given time $t$, as a scalar variable $D^{t}$ verifying the following conditions:

$$
\begin{aligned}
& \text { (i) } D^{t}=D^{t}\left(d^{t}\right) \\
& \text { (ii) } 0 \leq D^{t} \leq 1 \\
& \text { (iii) } D^{t}=0 \Longleftrightarrow d^{t}=0 \text { for all points } \\
& \\
& D^{t}=1 \Longleftrightarrow d^{t}=1 \text { for all points } \\
& \text { (iv) } D^{t_{1} \leq D^{t_{2}} \Longleftrightarrow t_{1} \leq t_{2}}
\end{aligned}
$$


Among the many possibilities for the definition of the 'global damage index' we propose the following two:

$$
\begin{aligned}
& D_{1}^{t}=\frac{\int_{\Omega} d^{t} d \Omega}{\int_{\Omega} d \Omega} \\
& D_{2}^{t}=\frac{\sqrt{\int_{\Omega}\left(d^{t}\right)^{2} d \Omega}}{\sqrt{\int_{\Omega} d \Omega}}
\end{aligned}
$$

where $d^{t}$ is the local damage variable. Note that both definitions verify the necessary conditions.

The first proposed alternative $D_{1}^{t}$ is the average value of the local damage over the nonlinear part of the structure and it is the simplest measure of global damage that can be devised. However, this value does not consider at all the distribution of damage over the structure. In particular, it is not sensitive to the existance of relatively small areas with relatively high values of damage, a circumstance that can very well affect significantly the overall behaviour of the dam. On the other hand, the second proposal $\dot{D}_{2}^{t}$ gives a greater weight to the higher values of local damage, and thus it takes somehow into account the localization effect. For the same value of the average damage $D_{1}^{t}$, the index $D_{2}^{t}$ will be greater depending on the degree of localization of the damage variable. That is why it has some interest the consideration of both indexes when comparing different distributions of local damage for the same structure subjected to different seismic excitations.

\subsection{Definition of the structural collapse}

When attempting to perform the safety assessment of a concrete dam against seismic actions it is important to define a situation of structural collapse. Ideally, this definition should be unambiguous and independent of the computational model used to analize the structural behaviour. Furthermore, the safety assessment must answer to (at least) three quite different questions: firstly, does the dam collapse while subjected to an earthquake of a given intensity and with a given response spectrum?; secondly, if this is not the case, which is the state of the dam after the seismic excititation?; and thirdly, how has the factor of safety of the dam against, for instance, the hydrostatic pressure been affected by the action of the earthquake?.

In this work we will distinguish between:

- Dynamic collapse during the earthquake, in any of the three following related situations: (a) when the computed displacements at selected points reach unaceptable values or grow unbounded, or (b) when the state of damage is either widely spread or highly localized, or (c) when the non-linear dynamic response departs greatly from the one obtained in a linear analysis.

- Static collapse after the earthquake, when the dam is able to withstand the seismic excitation but a quasi-static analysis performed a posteriori shows that the factor of safety against the action of the hydrostatic pressure has been greatly reduced due to the state of degradation induced by the earthquake. 
It is clear that these definitions are not totally objective, and more effort should be put into their quantitative assessment, particularly for the dynamic collapse. Also, they are linked somehow to the computational model used in the analysis (a damage model in this case). However, they adress questions of engineering interest and could be 'easily' re-interpreted for other material models, such as plasțicity or smeared crack models or fracture mechanics.

\section{SEISMIC ANALYSIS OF A GRAVITY DAM}

The computational model described the previous sections is now applied to the analysis of a concrete gravity dam. The geometry of the problem is depicted in Figure 1. The dam selected resembles very closely Koyna Dam in India (107 $\mathrm{m}$ high) that has been studied by many researchers interested in seismic analysis. The water level in the reservoir is $100 \mathrm{~m}$. The natural damping is tuned to provide a damping ratio of $5 \%$ for the first and second modes of the dam-soil system (Rayleigh parameters $a=0.8 \mathrm{~s}^{-1}, b=0.003 \mathrm{~s}$ ).

With the objective of analysing the response of this large concrete dam subjected to seismic actions of different intensities an artificial velocigram has been generated ${ }^{12}$. The design spectrum and the response espectrum of the generated accelerogram are compared in Figure 4, which shows the good agreement obtained. The generated velocigram has a total duration of 10 seconds, with a peak ground acceleration of $0.255 \mathrm{~g}$ and maximum ground velocities around $0.25 \mathrm{~m} / \mathrm{s}$ (occurring about $t=3.7 \mathrm{~s}$. The dominant period of the signal is $0.4 s$, which is very close to the first fundamental period of the dam-soil system $\left(T_{1}=0.4355 \mathrm{~s}\right)$. This input velocigram is shown in Figure 5 compared with the velocity computed at the free-field of the computational domain. Note that, apart for the shift due to the time that the signal takes to travel though the soil domain, the agreement between both of them is good. This shows that the boundary conditions are properly represented in the computational model.

Three seismic analyses are performed by scaling the input velocigram to achieve peak ground accelerations of $0.255 g, 0.383 g$ and $0.510 g$, respectively. The corresponding signals are applied as an horizontal seismic excitation along the canyon direction. Figure 6 shows the computed horizontal displacements (relative to the ground) for the three signals. Nonlinear effects are present for the smallest intensity, and grow significantly for the other two (stronger) signals. This is also evident in Figure 7, which depicts the evolution with time of the global (tension) damage index $D_{2}^{t}$ (no compression damage occurred during any of the analyses). Note that the evolution of damage is mostly concentrated in a few intervals of time where the maximum positive and negative displacements occur (close to $t=1.6 \mathrm{~s}$. and $t=4.0 \mathrm{~s}$.). This is again shown in Figures 8 and 9 , which show the distribution of damage and the deformed shape of the dam at selected times for the lowest and medium intensities, respectively. Note how for $0.255 \mathrm{~g}$ damage does not bridge across the 'neck' of the dam, whereas this does occur for $0.383 \mathrm{~g}$. However, as the up-stream and down-stream faces are not under tensile stress at the same time, the dam retains its overall stability. Regarding the evolution of the hydrodynamic pressure acting on the up-stream wall (not shown), it must be said that the graphs follow closely those of the (relative) horizontal displacement. Negative pressures are only encountered in the upper tenth of the dam, for 


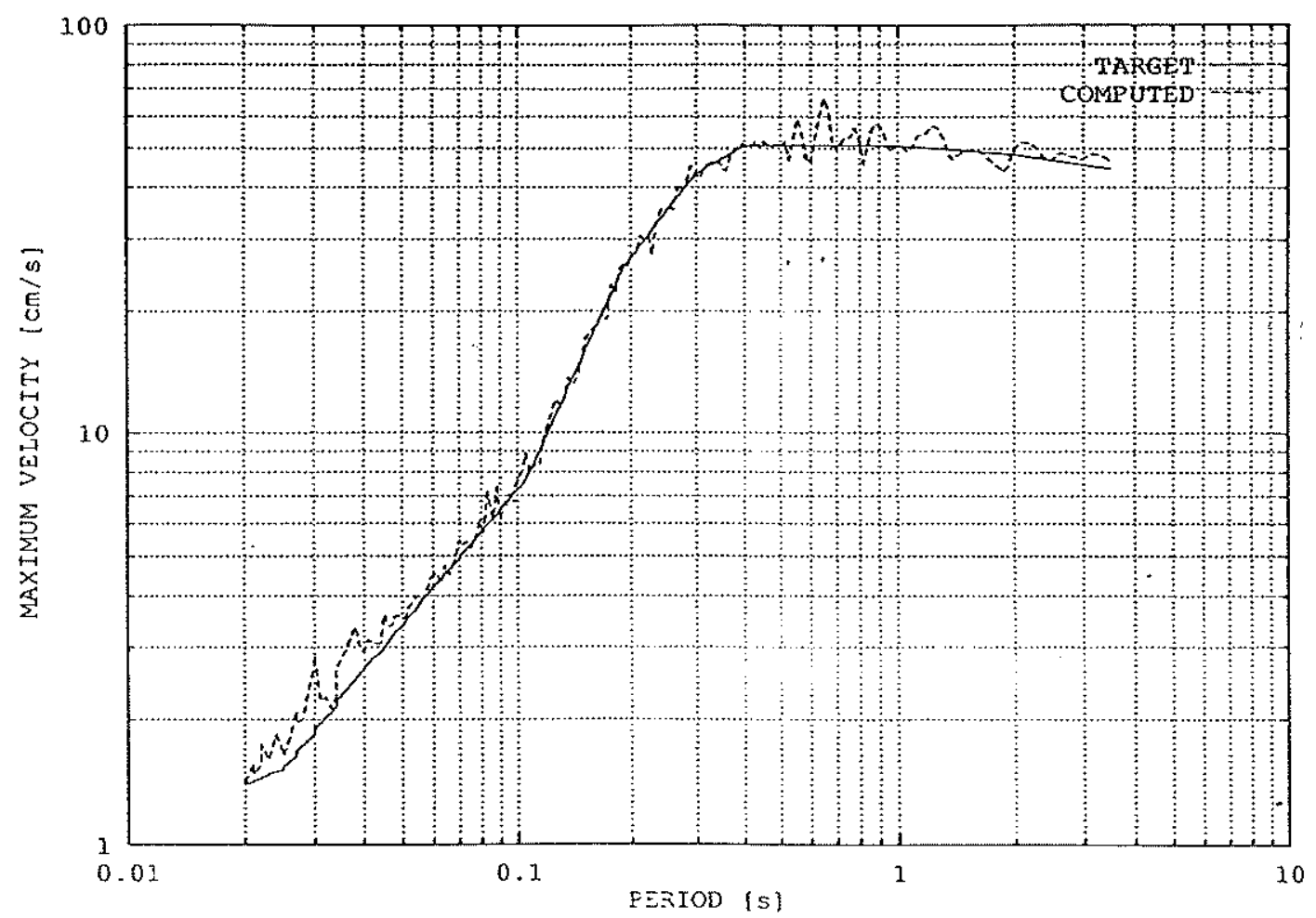

Figure 4. Comparison between the design and the computed velocity spectrum

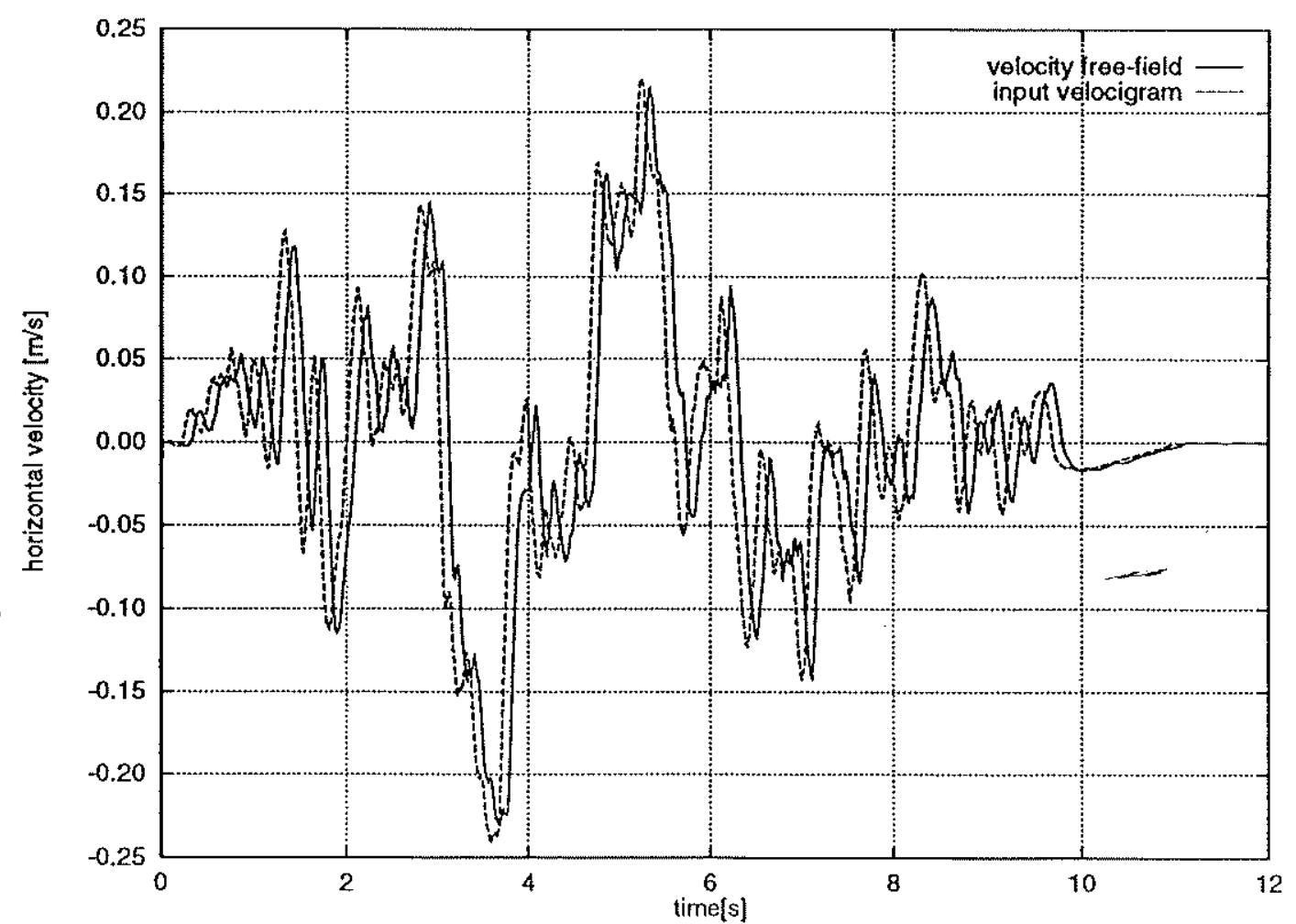

Figure 5. Comparison between the input velocigram and the computed velocity at the free-field 


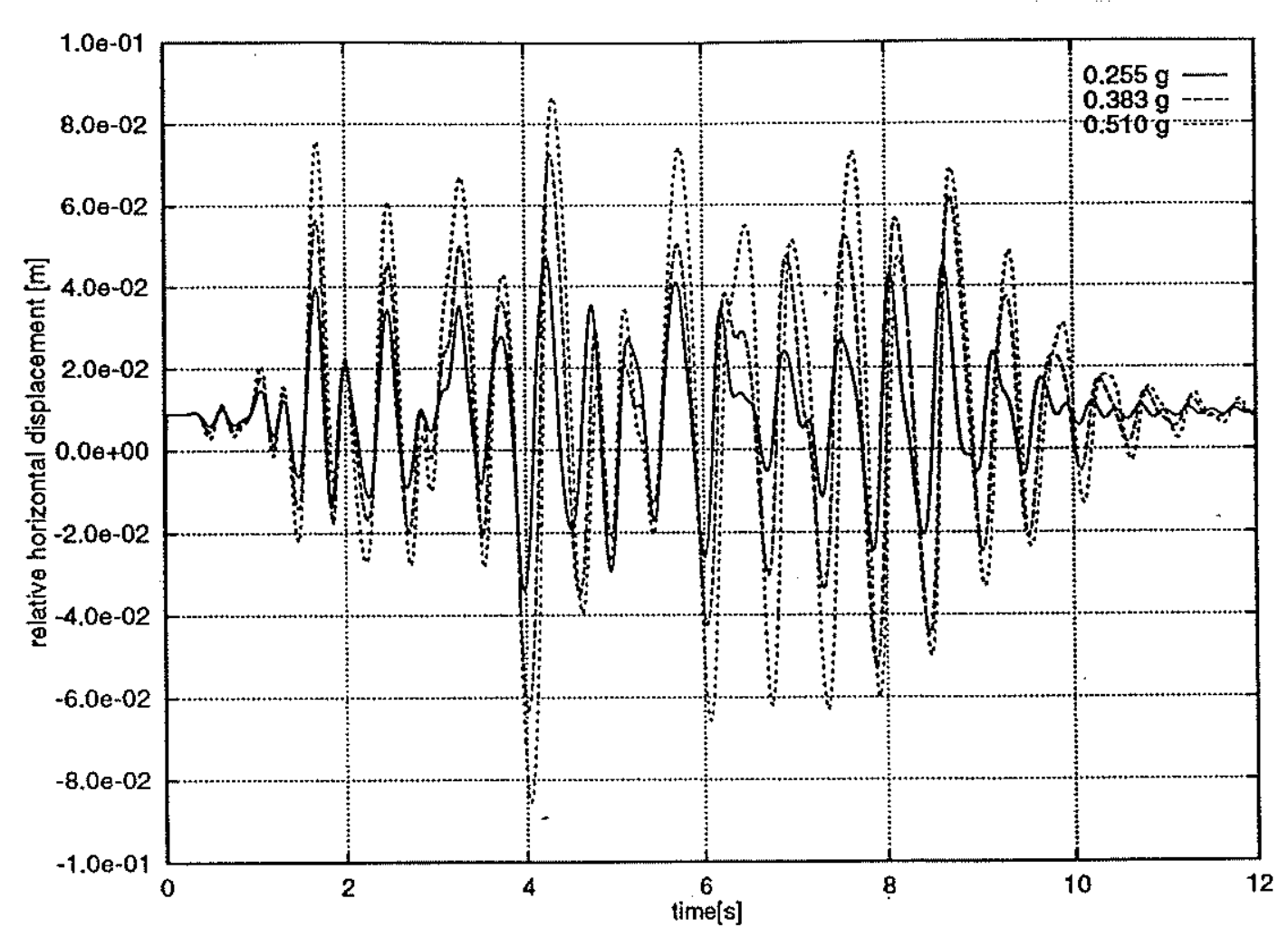

Figure 6. Koyna Dam. Horizontal (relative) displacement at the top of the dam for different seismic intensities

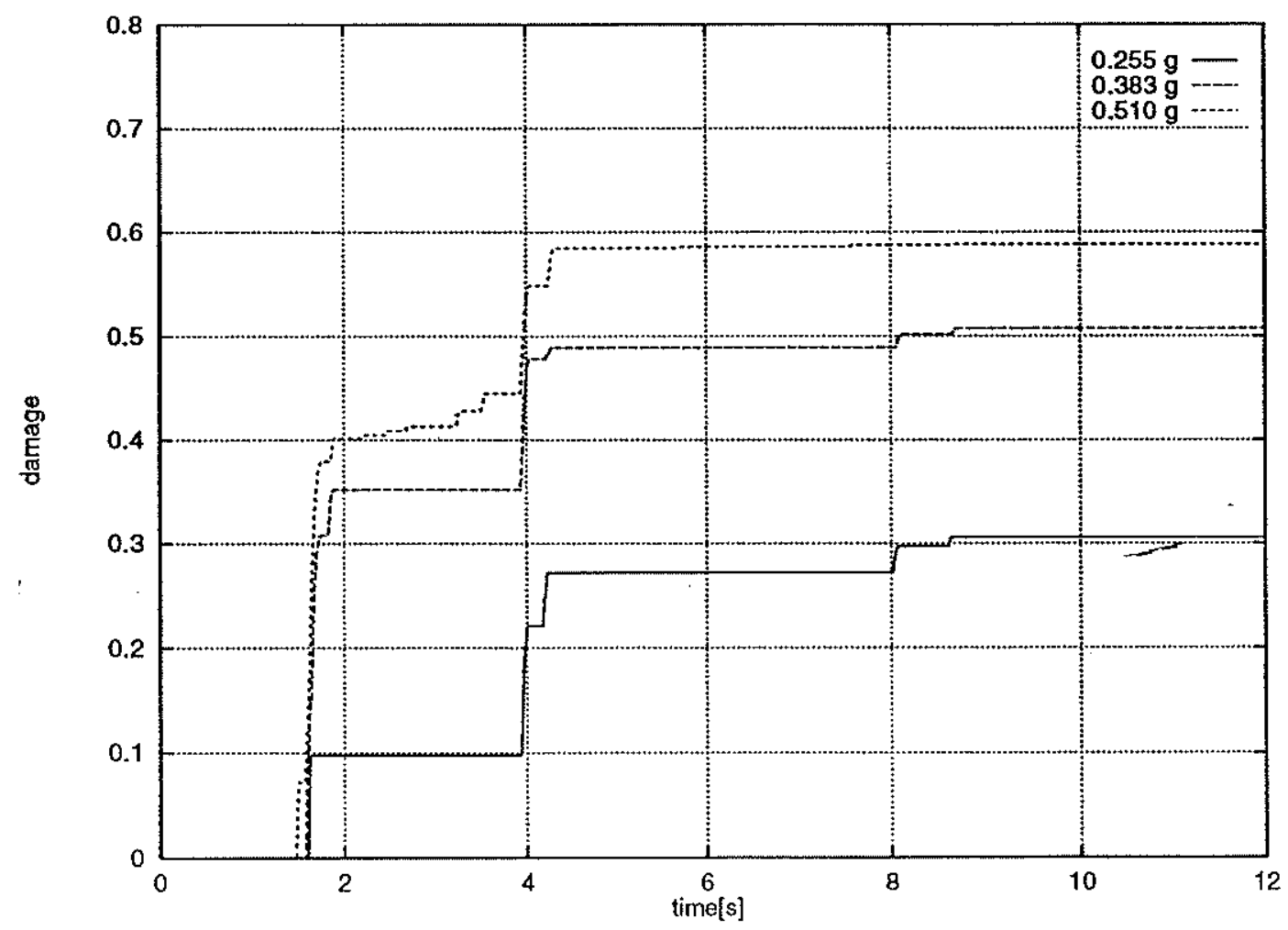

Figure 7. Koyna Dam. Evolution of the global (mean square value) tensile damage index for different seismic intensities 


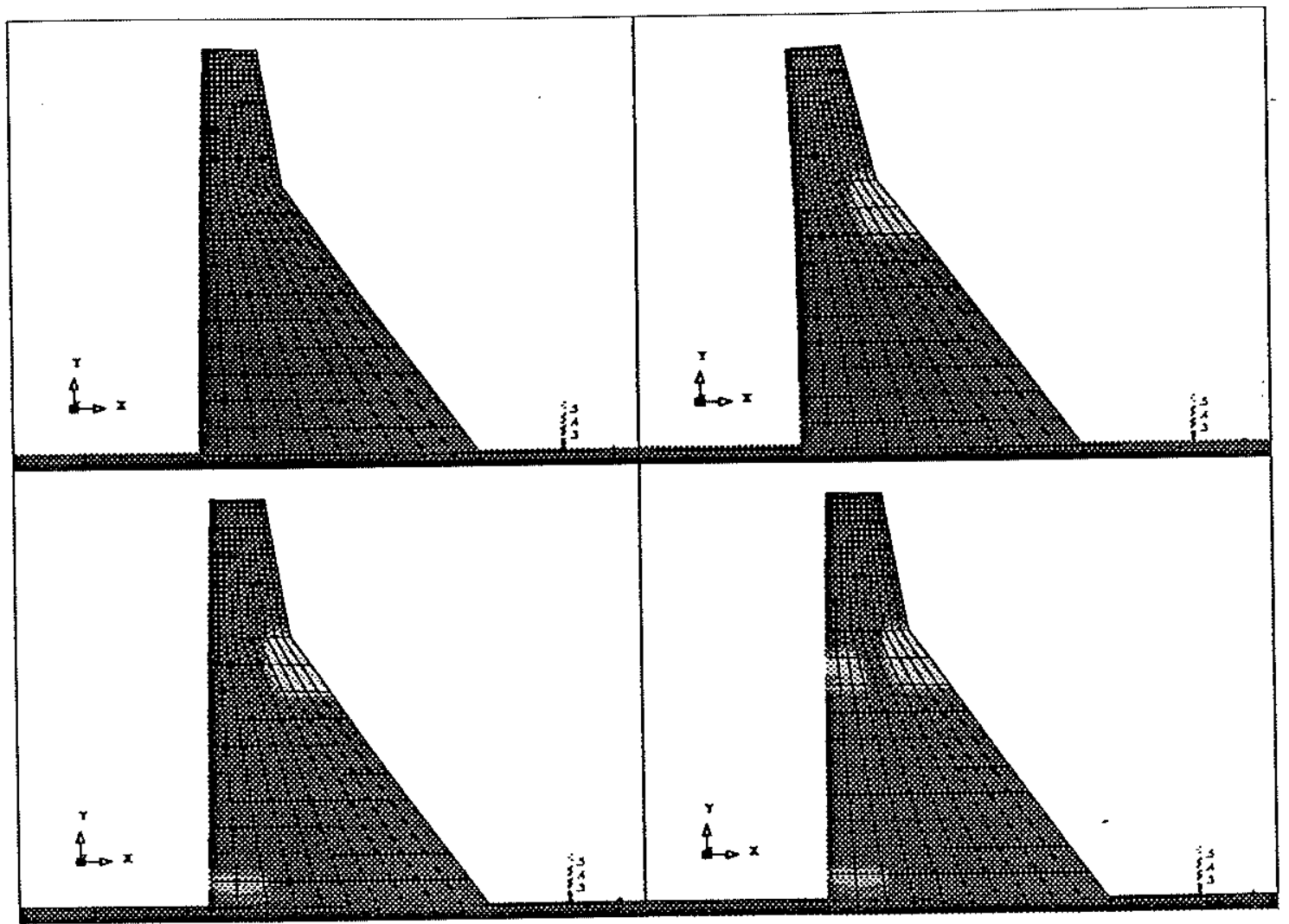

Figure 8. Koyna Dam. Evolution of the tensile damage and deformed shapes for the $0.255 \mathrm{~g}$ signal

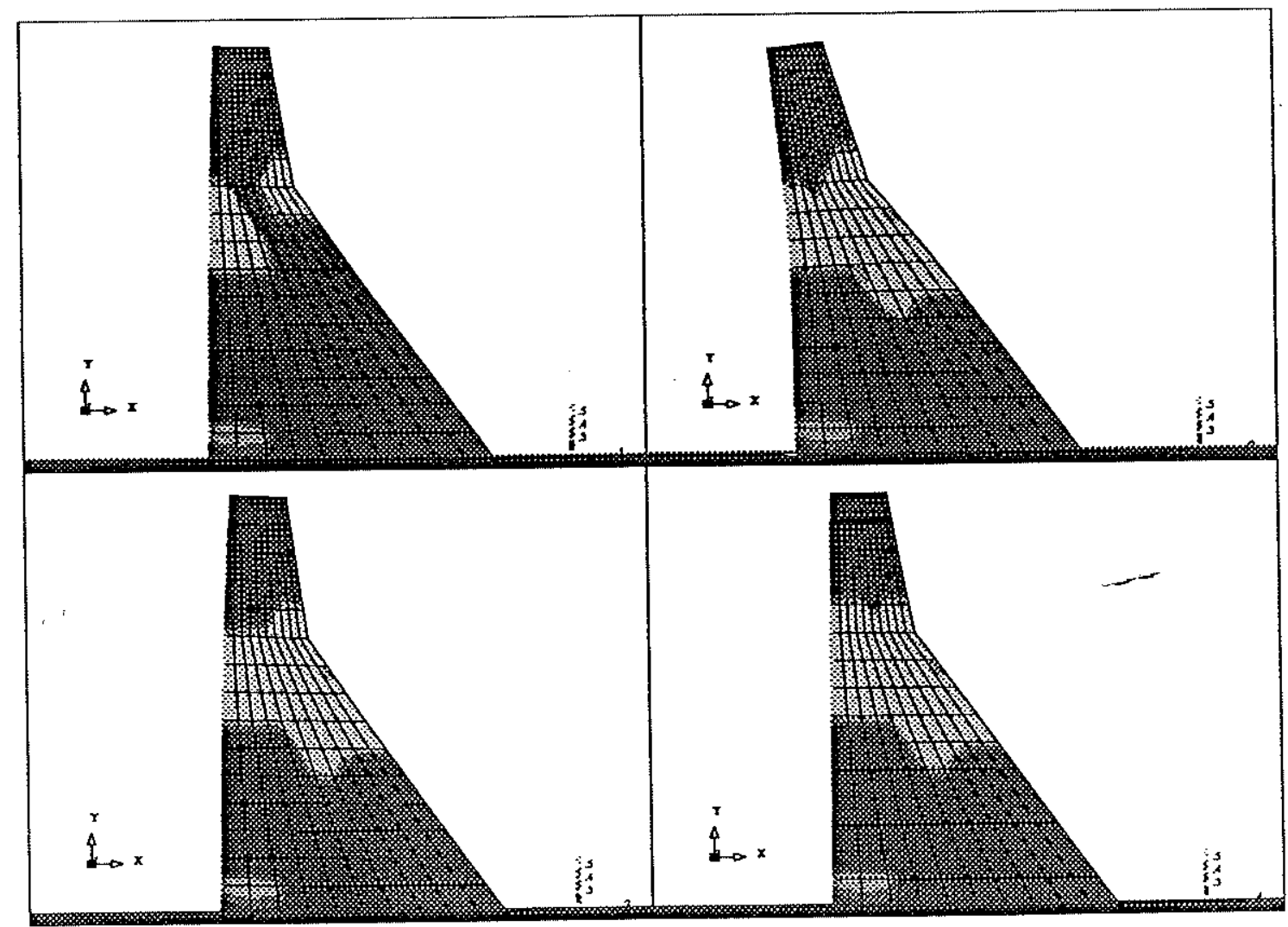

Figure 9. Koyna Dam. Evolution of the tensile damage and deformed shapes for the $0.383 g$ signal 
the highest intensity and only for $t=4.0 \mathrm{~s}$. Thus, it seems that the assumption of linear behaviour for the fluid is valid for this particular case.

About the assessment of seismic safety the results of the analyses are open to discussion. It can be said that the dam is severely damaged by the earthquake with a peak acceleration of $0.255 \mathrm{~g}$, and more importantly for the stronger signals. Therefore, the collapse intensity for a signal with this particular frequency content could be placed around $0.250 \mathrm{~g}$. However, it must be remarked that in all the analyses the dam retains its structural integrity. Furthermore, the a posteriori quasi-static analyses with increasing hydrostatic pressure produce a safety factor of 2.28 for all cases, with an identical collapse mechanism of over-turning. This can be explained considering that a gravity dam resists the hydrostatic pressure due to its self-weight, and for this load it is subjected to a triaxial state of compression everywhere. Clearly, this structural mechanism is not affected at all by the tensile damage induced by the earthquake.

\section{SEISMIC ANALYSIS OF AN ARCH DAM}

The computational model described in the previous sections is now applied to a the seismic analysis of an arch dam, which contitutes a $3 \mathrm{D}$ nonlinear coupled problem. To this end we have selected Talvacchia Dam, in Italy, a 75 meters high double curvature dam which has been studied previously by several researchers. The finite element meshes used for the reservoir water and the dam-soil domain are shown in Figure 10. It must be remarked than although the Figure shows both meshes together, the analysis has been performed separatedly for each of the domains, following the scheme presented in Section 3. Damping elements are appropriately situated at the bottom and lateral boundaries to prevent the out-going waves from artificially reflecting on them. The model is subjected to a horizontal earthquake in the longitudinal direction of the river (symmetric excitation), propagating vertically from the base of the model.

Figure 11 shows the amplified deformed shape of the dam (amplification factor equal to 500) for two given times (top and bottom) of the analysis correspondent to the earthquake with a peak acceleration of $0.364 \mathrm{~g}$. The Figure shows only half of the model (the water is not shown for clarity) from the front (left) and from the rear (right), with the corresponding tensile iso-damage contours. As expected, due to the symmetry of the model and the excitation, the higher values of the damage appear at the top of the central cantilever. Lower values of damage appear at the dam-foundation interface in the down-stream wall. Figure 12 shows the horizontal displacement at the top of the central cantilever for different intensity factors of the excitation. It can be seen that the response for a peak acceleration of $0.312 g$ is only mildly nonlinear, while for $0.364 g$, and specially for $0.416 g$, the nonlinear effects are evident. Figure 13 shows the evolution of the global (mean square value) damage with time for these three intensities. It can be seen that most of the damage develop during the first two seconds of the excitation, where the peak accelerations of the ground occur. Note that, nevertheless, important displacements occur later without changing significantly the level of damage.

Regarding the computational cost of the analysis it must be said that the additional cost due to the fluid interaction is very small (about $4 \%$ of the total CPU time), because 
of the formulation used (a single degree of freedom per node). Unfortunately, most of the computer time is spent by the foundation; therefore, and always supposing that linear behaviour is a valid assumption, alternative techniques, such as the boundary element method, may be used. In this work, we have used reduced integration with an hourglasscontrol technique to keep the computational cost within reasonable limits.

\section{CONCLUSIONS}

The paper presents a general methodology for the evaluation of large dams subjected to seismic actions with two main objectives: generality, as it is valid both for gravity and arch dams and it includes most of the relevant features present in the problem, and efficiency, as it allows for the use of modular and computationally efficient software. Four different conclusions can be drawn from this work: (a) The non-linear behaviour of the dam may be appropriately represented via an isotropic continuum damage model with separated internal variables for degradation under tension and compression stress states. Moreover, this option allows for stiffness recovery (also known as unilateral effect) upon load reversal, a feature of primary importance for seismic analysis of structures. Also, the model is particularly suitable for large scale problems due to its explicit format. (b) The treatment of the boundary conditions for the dynamic transient problem is fully stated both for the solid and the fluid phases in the computational model. The introduction of radiating or 'transparent' boundaries, and the imposition of the in-coming seismic wave as an appropriate force term seem an adecuate option from the analytical and computational standpoints. (c) The partitioned block-iterative solution of the fluid-structure interaction problem is formulated and tested for real application analyses. It is found that both the non-linearity and the coupling can be treated most efficiently within a single iterative loop, without loss of generality. No stability difficulties are found for real case data and the overhead to be paid due to the coupled analysis of the fluid is almost negligible. (d) Finally, some qualitative criteria for the definition of structural collapse and the seismic assessment of concrete dams are presented. The topic is open for discussion and future work must be primarily directed in this direction. To conclude, the application of the proposed methodology to the seismic analysis of two real dams of different topologies shows that it can be used as a valid tool for earthquake engineering and structural analysis. 


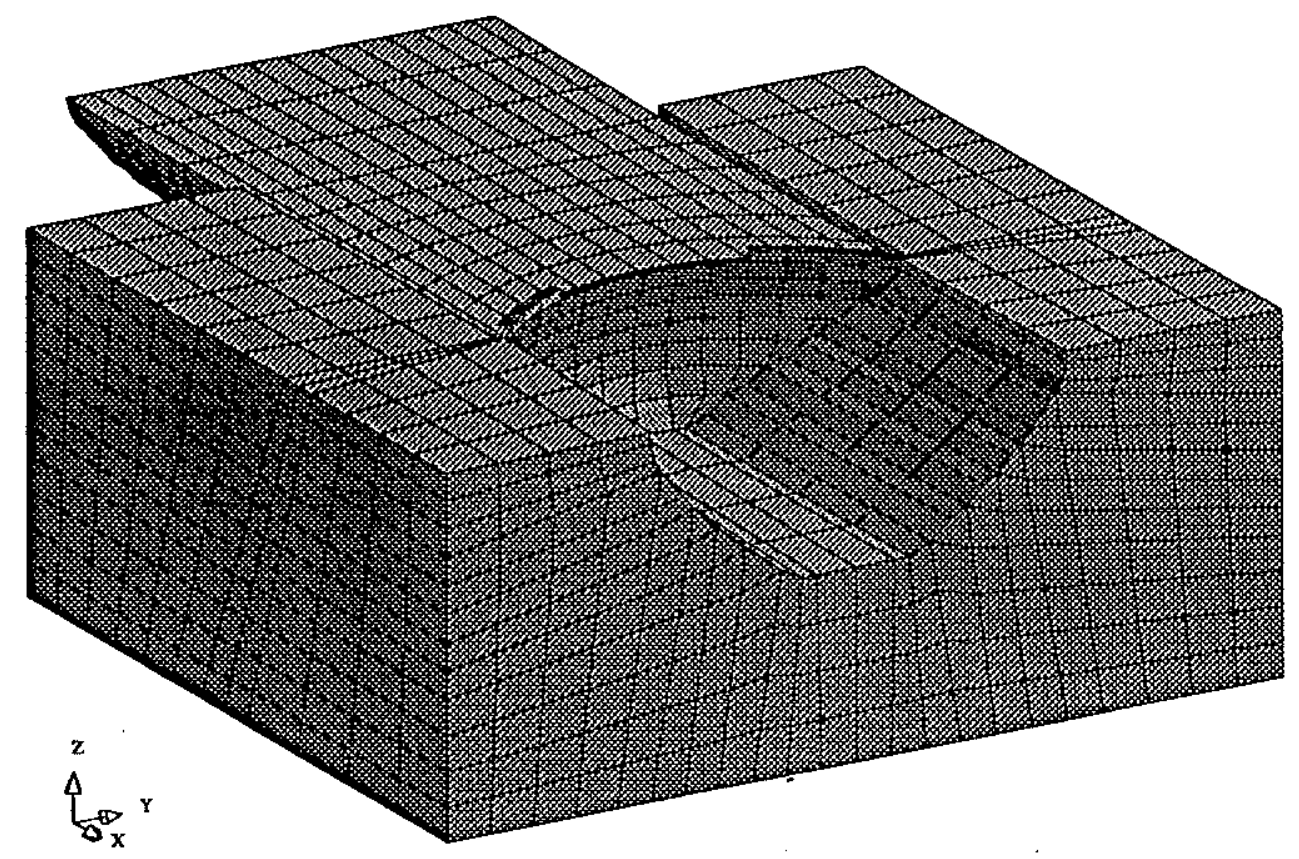

Figure 10. Talvacchia Dam. Computational model

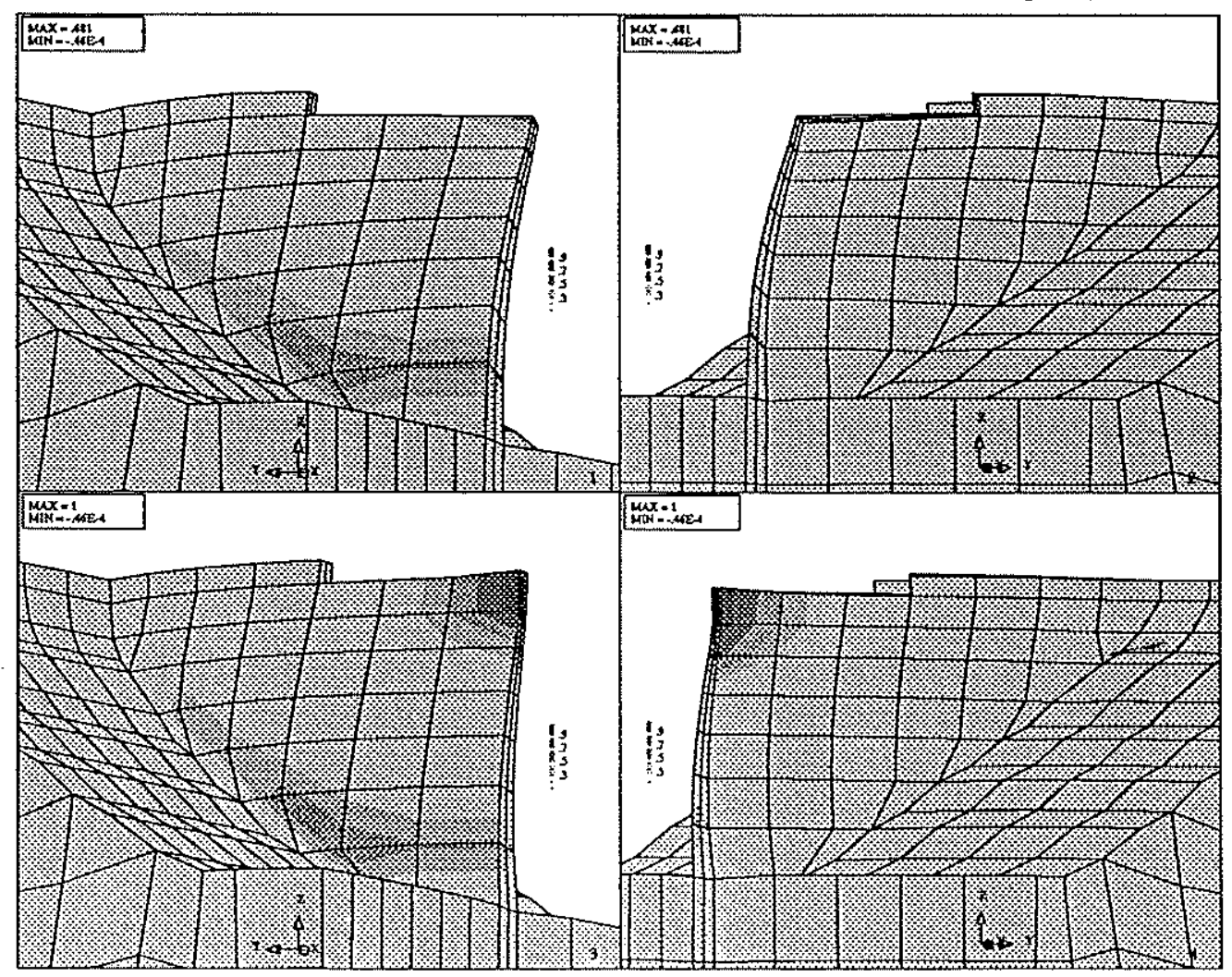

Figure 11. Talvacchia Dam. Deformed mesh with iso-damage contours 


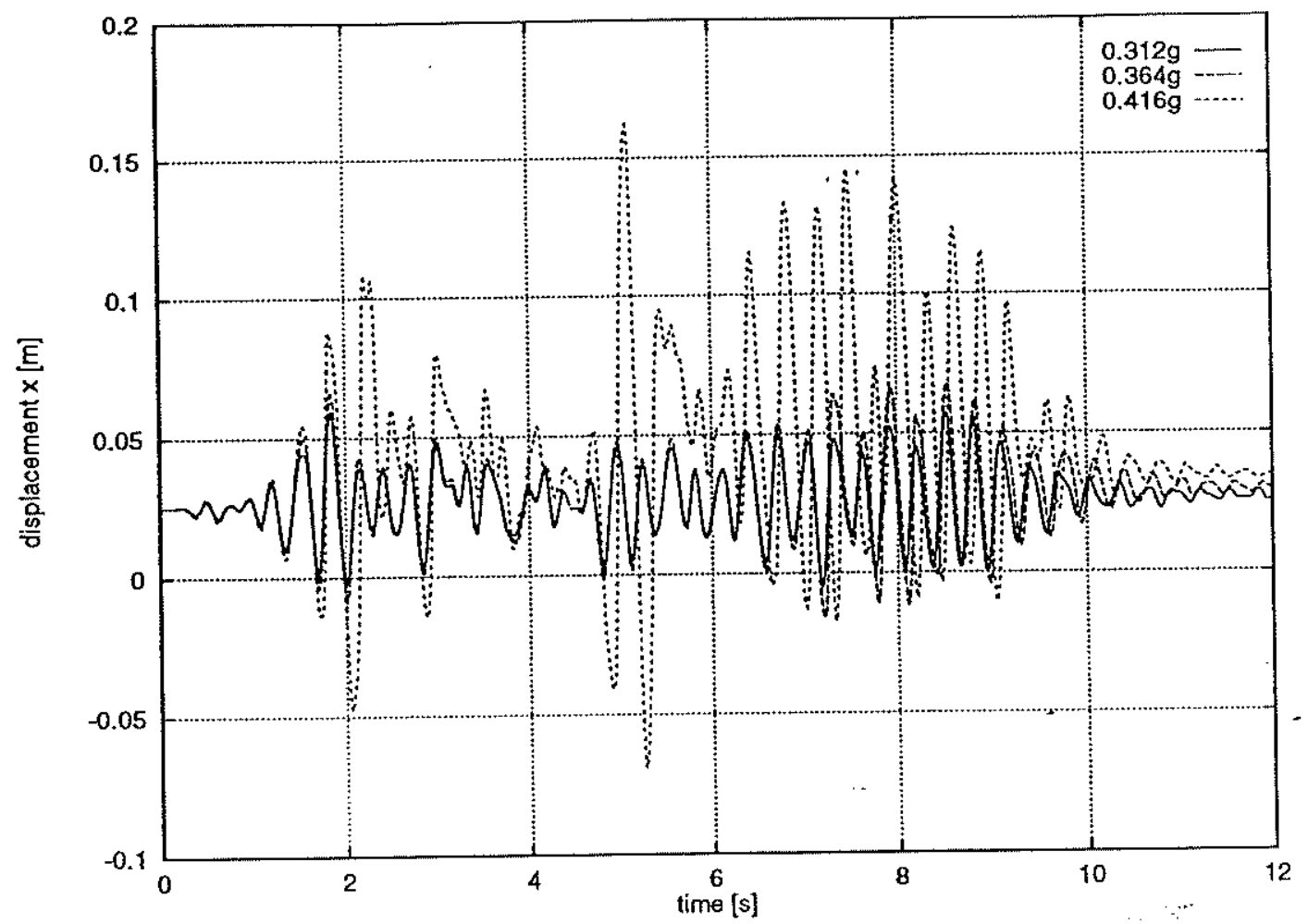

Figure 12. Talvacchia Dam. Horizontal displacement at the top of the central cantilever for different intensity factors

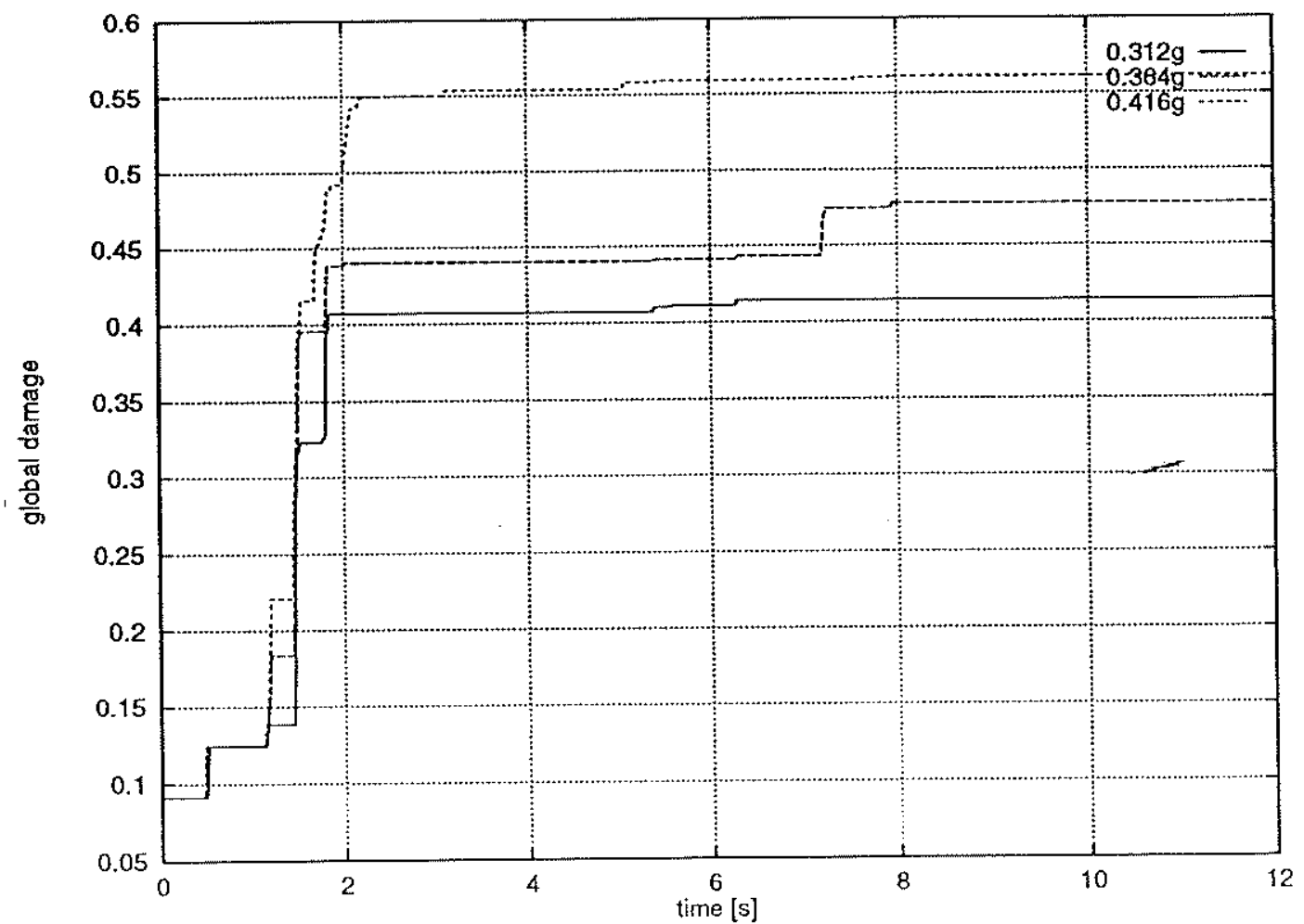

Figure 13. Talvacchia Dam. Evolution of the global (mean square value) damage for different intensity factors 


\section{REFERENCES}

1 L. M. Kachanov, 'Time of rupture process under creep conditions'. Izvestia Akademii Nauk, Otd Tech Nauk., 8, 26-31 (1958).

2 J. Lemaitre and J. L. Chaboche, 'Aspects Phénoménologiques de la Rupture par Endommagement'. J. Méc. Appl., Vol. 2, 3, 317-365 (1978).

3 J. Lemaitre, 'How to use Damage Mechanics'. Nuclear Eng. and Design, 80, 233-245 (1984).

4 J. C. Simo and J. W. Ju, 'Strain- and Stress-Based Continuum Damage Models - I. Formulation'. Int. J. Solids Structures, Vol. 23, 7, 821-840 (1987).

5 J. C. Simo and J. W. Ju, 'Strain- and Stress-Based Continuum Damage Models - II. Computational aspects'. Int. J. Solids Structures, Vol. 23, 7, 841-869 (1987).

6 J. L. Chaboche, 'Continuum Damage Mechanics: Part I - General Concepts'. J. Appl. Mechanics, 55, 59-64 (1988).

7 J. L. Chaboche, 'Continuum Damage Mechanics: Part II - Damage Growth'. J. Appl. Mechanics, 55, 65-72 (1988).

8 J. Mazars and G. Pijaudier-Cabot, 'Continuum Damage Theory - Application to Concrete'. J. of Eng. Mech., ASCE, Vol. 115, 2, 345-365 (1989).

9 J. Oliver, M. Cervera, S. Oller and J. Lubliner," Isotropic Damage Models and Smeared Crack Analysis of Concrete'. Proc. 2nd Int. Conf. on Computer Aided Analysis and Design of Concrete Structures., 945-957, Pineridge Press (1990).

10 C. A. Felippa and K. C. Park, 'Staggered transient analysis procedures for coupled mechanical systems: Formulation'. Com. Meth. Appl. Mech. and Engng., 24, 61-111 (1980).

11 D. K. Paul, Efficient Dynamic Solutions for Single and Coupled Multiple Field Problems. Doctoral Thesis, University of Wales (1982).

12 M. Galindo, Una metodología para el análisis numérico del comportamiento resistente no lineal de presas de hormigón con cargas estáticas y dinámicas . Doctoral Thesis, Technical University of Catalonia (1993).

13 R. Codina, M. Cervera and M. Galindo, 'On the efficiency of block-iterative algorithms for two nonlinear coupled problems', Proceedings of the II Asian-Pacific Conference on Computational Mechanics, 1241-1246, Balkema (1993).

14 M. Galindo, M. Cervera and J. Oliver, 'Efficient solution schemes for fluid-structure-soil interaction problems', Proceedings of 10th World Congress on Earthquake Engineering, 4651-4656, Balkema (1991).

15 O. Z. Zienkiewicz and R. L. Taylor, The finite element method. McGraw Hill (1991).

16 T. J. R. Hughes, The Finite Element Method: Linear Static and Dynamic Finite Element Analysis. Prentice-Hall International (1987).

17 T. J. R. Hughes, K. S. Pister and R. L. Taylor, 'Implicit-explicit finite elements in nonlinear transient analysis'. Comp. Meth. Appl. Mech. Engng., 17/18, 159-182 (1979).

18 S. S. Bhattacharjee and P. Léger, 'Seismic cracking analysis and energy dissipation in concrete gravity dams.' Earth. Engng. Struc. Dyn., 22, 991-1007 (1980).

19 R. Faria and J. Oliver, A Rate Dependent Plastic-Damage Constitutive Model for Large Scale Computations in Concrete Structures. Monography CIMNE, N. 17. (1993). 
20 M. Galindo, M. Cervera and J. Oliver, 'Parallel Synchonized Communication: an algorithmic approach to coupled problems'. Proceedings of the International Conference on Numerical Methods in Engineering and Applied Sciences, 389-398, CIMNE (1992).

21 O. C. Zienkiewicz, D. K. Paul and A. H. C. Chan, 'Unconditionally stable staggered solution procedures for soil-pore fluid interaction analysis'. Int. J. Num. Meth. Eng., 19, 1669-1673 (1986).

22 A. H. C. Chan, $A$ unified Finite Element Solution to Static and Dynamic problems of Geornechanics. Doctoral Thesis, University of Wales (1988).

23 K. Wisniewski, E. Turska, L. Simoni and B. A. Schrefler, 'Error analysis of staggered predictor-corrector scheme for consolidation porous media', The Finite Element Method in the 1990's. Springer-Verlag/CIMNE (1991).

24 M. Cervera, R. Codina and M. Galindo, On the computational efficiency and implementation of block-iterative algorithms for nonlinear coupled problems, Research Report CIMNE, N. 43. (1994).

25 O. C. Zienkiewicz, R. W. Clough and H. B. Seed, Eartquake analysis procedures for concrete and earth dams, Research Report INME, C/R/457/83 (1984).

26 M. Fanelli, 'The safety factor of dams - an abstract concept or a measurable quantity?'. Dam Engineering, Vol. II, 2, 97-99 (1991). 